Columbia Law School

Scholarship Archive

2020

\title{
Discrete Rent-Seeking Games with an Application to Evidence Production
}

Giuseppe Dari-Mattiacci

Columbia Law School

Lewis A. Kornhauser

New York University School of Law, lewis.kornhauser@nyu.edu

Follow this and additional works at: https://scholarship.law.columbia.edu/faculty_scholarship

Part of the Evidence Commons

\section{Recommended Citation}

Giuseppe Dari-Mattiacci \& Lewis A. Kornhauser, Discrete Rent-Seeking Games with an Application to Evidence Production, 28 Sup. CT. Econ. Rev. 285 (2020).

Available at: https://scholarship.law.columbia.edu/faculty_scholarship/3164

This Article is brought to you for free and open access by the Faculty Publications at Scholarship Archive. It has been accepted for inclusion in Faculty Scholarship by an authorized administrator of Scholarship Archive. For more information, please contact scholarshiparchive@law.columbia.edu, rwitt@law.columbia.edu. 


\title{
Discrete Rent-Seeking Games with an Application to Evidence Production
}

\author{
Giuseppe Dari-Mattiacci*
}

Lewis A. Kornhauser ${ }^{+}$

Evidence production at trial, the accumulation of patents in a technological race, and lobbying are contests that often involve strategic choices over a discrete set of options. The literature has primarily focused on games with continuous effort choices. We fill this gap by studying a rent-seeking game with discrete effort choices and, for a significant class of games, derive a transformation rule that allows one to find the equilibrium of the discrete game from the equilibrium of the continuous game, which is much simpler to identify. We also discuss the limits of this approach and how well the continuous game approximates the discrete one.

\section{INTRODUCTION}

Parties engaged in a legal dispute compete with each other in the production of evidence, which in turn determines the way in which the court will adjudicate the case. Evidence production can be studied through the lens of rent-seeking games: the parties' expended efforts are otherwise unproductive investments whose only purpose is to affect the way in which the "pie" will be divided by the court. Although the amount and quality of the evidence produced by the parties can have beneficial effects for society in terms of better-quality decisions,

* Giuseppe Dari-Mattiacci is the Alfred W. Bressler Professor of Law at Columbia Law School.

† Lewis A. Kornhauser is the Frank Henry Sommer Professor of Law at New York University. The authors would like to thank the editor Murat Mungan and an anonymous referee for very helpful comments. 
the evolution of new precedents, and the production of ex ante incentives to avoid harmful behavior-and hence evidence production is not at all a worthless activity-the rent-seeking perspective captures fairly accurately the particular incentives of most parties ex post, that is, at the moment they become involved in a dispute.

There is a vast literature on rent-seeking games-originating from the seminal papers by Tullock $(1967,1980)$ and Krueger $(1974)$-and this literature has examined virtually all possible variations of the original setting. ${ }^{1}$ However, the universal approach has been to consider the effort expended by the contestants as a continuous variable. ${ }^{2}$ Indeed, monetary expenditures can be made in increments that are small enough to be approximated by a continuous variable. Nevertheless, in many real-life situations, the parties can only choose among a discrete (albeit possibly infinite) set of effort levels. Evidence production, for instance, involves discrete decisions, such as the number of witnesses to appear in court or how many experts to hire. Many other rent-seeking contexts also involve discrete decisions. Think of a race to accumulate patents related to a particular technology or the decision to hire an additional lobbyist. In this article, we fill this gap by considering the classic rent-seeking game in which two (possibly asymmetric) risk-neutral parties seek an indivisible prize; the investment of each party determines the probability that each will win. (Given risk-neutrality, this setup also captures cases in which the prize is split between the parties.) Differently from the standard setup, the parties make a discrete number of investments into the game.

We analyze a class of symmetric and asymmetric rent-seeking games with discrete strategy spaces and address several questions. Our main result is presented in proposition 1 . We identify a sufficient condition under which the equilibrium of the discrete game lies in the closest possible neighborhood around the equilibrium of the continuous game. This condition essentially points to the asymmetry between the parties' costs of efforts as the relevant factor. If the parties' costs of effort are relatively similar, the equilibrium of the discrete game lies neatly in the closest possible neighborhood around the equilibrium of the continuous game. However, as the parties' costs start to diverge, so do the parties' abilities to optimize their behavior. The party with the largest cost of effort can effectively choose among a more restricted set

\footnotetext{
${ }^{1}$ For recent reviews, see Congleton, Hillman, and Konrad (2008a, 2008b); Congleton and Hillman (2015). A review of the literature on asymmetric rent-seeking games, which will form the basis for our model, can be found in Dari-Mattiacci and Parisi (2015).

${ }^{2}$ A solitary exception to this trend is Baye, Kovenock, and Vries (1994), which, however, only analyzes symmetric games and focuses on the problem of increasing returns to effort. We examine possibly asymmetric parties with constant returns to effort.
} 
of options as compared with the other party with lower cost of efforta fact that we will illustrate momentarily by means of an example. The asymmetry in the parties' costs of effort hence translates into an asymmetry in their ability to optimize and results in an advantage for the lower-cost party. It is that party's ability to exploit this advantage that breaks the correspondence between the equilibrium of the continuous game and that of the discrete game. Under the condition identified in proposition 1, we provide four additional sets of results.

We show that, although the continuous game always has a unique pure strategy equilibrium, the discrete game may have a unique pure strategy equilibrium or a mixed strategy equilibrium /which we characterize). Next, we compare the equilibrium of the discrete game with the equilibrium of the continuous game and offer a simple transformation rule that allows the analyst to solve the discrete game by first solving the (much simpler) continuous game and then applying our transformation rule to the result. Moreover, we show that the continuous approximation of a discrete game is more accurate if (i) the contested prize grows toward infinity, (ii) the parties' marginal costs of effort are closer to each other, or (iii) the set of admissible discrete values for the parties' efforts becomes denser-that is, the interval between two contiguous admissible values decreases.

Finally, we demonstrate that if the condition identified in proposition 1 is not satisfied, the correspondence between the continuous and the discrete game may break down. This shows that, although the condition in proposition 1 is only sufficient, and hence we may err on the side of caution when using the equilibrium of a continuous game to approximate the equilibrium of the corresponding discrete game, caution is warranted because the procedure we propose applies only to a subset of games.

Next, we bring our theory to bear on a concrete contest in which parties make discrete investments into the game: evidence production in court. Typically, evidence is a collection of discrete elements: documents, experts, eyewitnesses, and so forth. In the model, parties hire an attorney at an hourly fee; the attorney then looks for evidence. We show that, although the attorney is paid in an (almost) continuous currency, the parties' strategy spaces remain discrete and the equilibrium can be found by using our transformation rule.

This article is organized as follows. In section 2, we present the basic model, discuss the benchmark case of the continuous game, and introduce the discrete game. In section 3, we study the discrete game and deliver our main results. In section 4 , we discuss the accuracy of the continuous approximation of the discrete game. In section 5 , we illustrate our findings in the context of a symmetric game. In section 6, we apply our theory to evidence production. Finally, in section 7, we conclude. 


\section{BASIC MODEL}

\subsection{Setup}

In the model, we use a standard framework à la Tullock. Two parties, $\mathrm{A}$ and $\mathrm{B}$, exert effort $a \geq 0$ and $b \geq 0$, respectively, in a contest to win a prize with value $V>0$. The prize is shared proportionally to the parties' efforts (and is shared equally if both parties invest 0 ). The parties are identical but for their marginal costs of effort, which are respectively $c_{\mathrm{A}}>0$ and $c_{\mathrm{B}}>0$.

Parties are risk-neutral and hence the success function can be interpreted indifferently as an actual share of the prize or as a probability of winning the entire prize. The parties' maximization problems are as follows:

$$
\begin{gathered}
\max _{a}\left[\frac{a}{a+b} V-c_{\mathrm{A}} a\right] \\
\max _{b}\left[\frac{b}{a+b} V-c_{\mathrm{B}} b\right]
\end{gathered}
$$

where we impose $a /(a+b)=b /(a+b)=1 / 2$ if $a=b=0$. In the next subsection, we review the standard case in which effort is a continuous variable, which we use as a benchmark. Next, we examine a discrete version of the same game.

\subsection{Benchmark: The Continuous Game}

As a benchmark, we consider the standard rent-seeking game with continuous effort spaces. Parties A and B play the game by choosing effort from the nonnegative real line: $a \in[0, \infty)$ and $b \in[0, \infty)$. The problems in equation (1) have first-order conditions $v b /(a+b)^{2}=c_{\mathrm{A}}$ and $v a /$ $(a+b)^{2}=c_{\mathrm{B}}$ for party $\mathrm{A}$ and $\mathrm{B}$, respectively. (The second-order conditions are satisfied.) As is well known, this game has a unique Nash equilibrium where the parties make the following investments into the game:

$$
\begin{aligned}
& a^{*}=\frac{c_{\mathrm{B}}}{\left(c_{\mathrm{A}}+c_{\mathrm{B}}\right)^{2}} V(\mathrm{~A}) \\
& b^{*}=\frac{c_{\mathrm{A}}}{\left(c_{\mathrm{A}}+c_{\mathrm{B}}\right)^{2}} V(\mathrm{~B})
\end{aligned}
$$

From the first-order conditions, we can derive the parties' reaction functions: 


$$
\begin{aligned}
& a^{* *}(b)=\sqrt{b \frac{V}{c_{\mathrm{A}}}}-b(\mathrm{~A}) \\
& b^{* *}(a)=\sqrt{a \frac{V}{c_{\mathrm{B}}}}-a(\mathrm{~B})
\end{aligned}
$$

which will be useful later.

\subsection{The Discrete Game}

Now let us consider an alternative model in which the parties' effort space is discrete: $a \in\{0, \varepsilon, 2 \varepsilon, 3 \varepsilon \ldots\}$ and $b \in\{0, \varepsilon, 2 \varepsilon, 3 \varepsilon \ldots\}$, where $\varepsilon>0$ is the distance between any two admissible levels of effort. We can think of $\varepsilon$ as a measure of the coarseness of the parties' choice sets. When $\varepsilon$ is large, the choice sets are coarse and the parties have relatively few options to choose among. In contrast, as $\varepsilon$ decreases, the parties' choice sets become denser, giving parties more opportunities to optimize their behavior. Note that as $\varepsilon \rightarrow 0$, the discrete game approaches the continuous game. (When $\varepsilon=1$, the parties' available efforts choices are nonnegative integers.)

With a slight abuse of terminology, we use the term "floor of $x$ " or the symbol $\underline{x}$ to refer to the element in the effort set $\{0, \varepsilon, 2 \varepsilon, 3 \varepsilon \ldots\}$ that is closest to the real number $x$ from below; analogously, we use the term "ceiling of $x^{\prime \prime}$ or the symbol $\bar{x}$ to refer to the closest value above $x$ in the set $\{0, \varepsilon, 2 \varepsilon, 3 \varepsilon \ldots\}$. (When $\varepsilon=1$, the floor of $x$ is the integer directly below $x$ and the ceiling of $x$ is the integer directly above $x$, as in the usual interpretation.) To visualize: in the continuous game, the parties' choices of effort can be represented by any point on the positive real plane with coordinates $(a, b)$. In contrast, in the discrete game, the parties' choices of efforts are restricted to the intersection points of a grid with square cells of width and height equal to $\varepsilon$, which lies in the same plane.

Note that, although the parties' effort sets are (countably) infinite, it is undesirable for a party to spend more than the prize at stake, so that the maximum effort a party can profitably make is $y_{i} \varepsilon=\left(\mathrm{V} / \mathrm{c}_{i}\right)$, for $i \in\{A, B\}$; that is, the greatest value in the set $\{0, \varepsilon, 2 \varepsilon, 3 \varepsilon \ldots\}$ that is less than $V / c_{i}$. In turn, this observation makes the parties' effort sets effectively finite, or equal to $\left\{0, \varepsilon, 2 \varepsilon, 3 \varepsilon, \ldots, y_{i} \varepsilon\right\}$. Application of Nash's (1951) theorem guarantees that the discrete game must have at least one Nash equilibrium in pure or mixed strategies.

To find the equilibrium of the discrete game in a simple way, we propose the following two-step procedure: 
1. Find the equilibrium of the continuous game, $\left(a^{*}, b^{*}\right)$;

2. Solve the restricted discrete game in which party A can only play $a \in\left\{\underline{a^{*}}, \overline{a^{*}}\right\}$ and party B can only play $b \in\left\{\underline{b^{*}}, \overline{b^{*}}\right\}$.

In section 3 , we will first show under what (sufficient, but not necessary) conditions the equilibrium of the restricted game is a (possibly unique) equilibrium of the full game. Next, we investigate whether the equilibrium of the continuous game is a good approximation of the equilibrium of the discrete game. Most of the proofs will leverage on the fact that a party's payoff function in equation (1) is strictly concave in that party's effort and hence parties prefer effort levels that are closer to their reaction functions.

\subsection{An Illustration}

Consider figure 1. The equilibrium of the continuous game lies at the intersection of the parties' reaction functions, the dashed lines in the

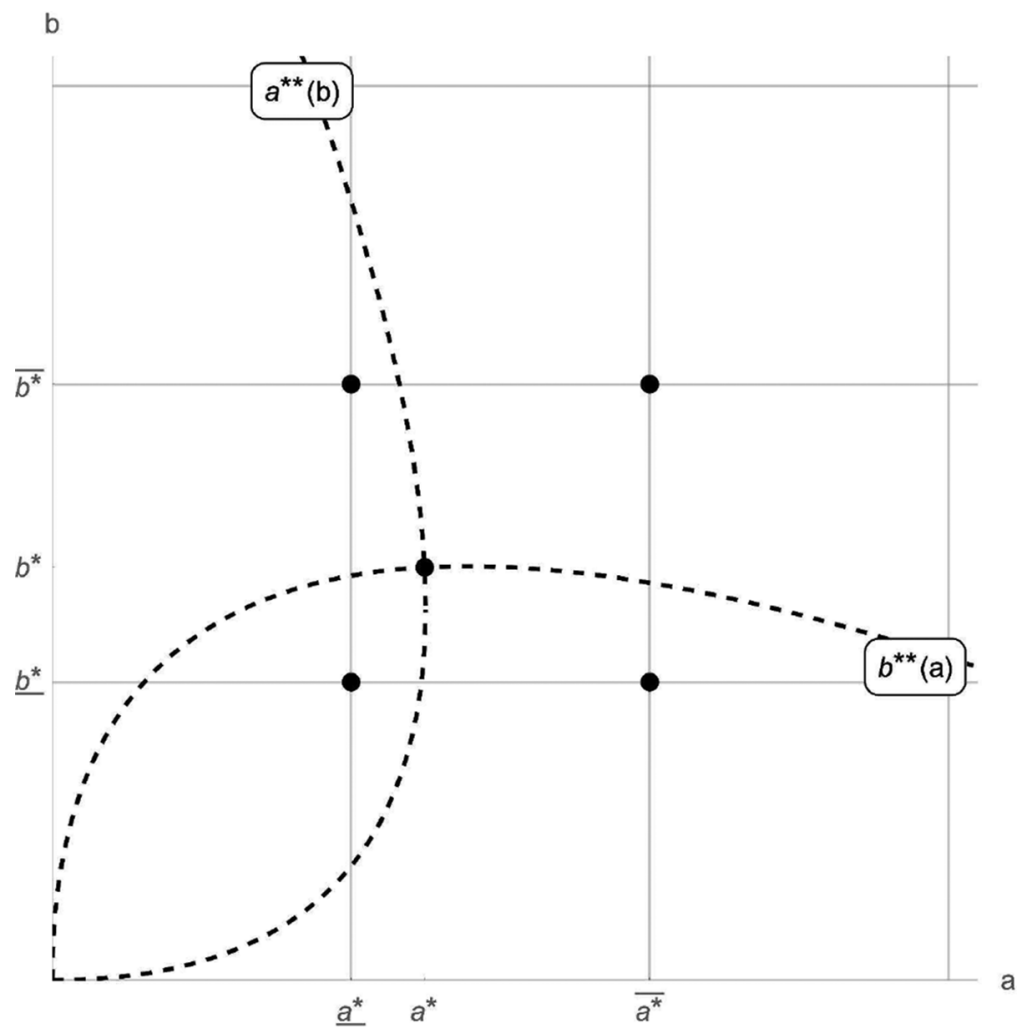

Figure 1. Candidate equilibria of a discrete game with $v=10, c_{A}=2, c_{B}=1.8$, and $\varepsilon=1$ 
Table 1. Parties' Payoffs $(A, B)$ in a Discrete Game with $v=10, c_{\mathrm{A}}=2$, $c_{\mathrm{B}}=1.8$ and $\varepsilon=1$

\begin{tabular}{c|llllll}
\hline$\ldots$ & $\ldots$ & \multicolumn{1}{c}{$\ldots$} & \multicolumn{1}{c}{$\ldots$} & \multicolumn{1}{c}{$\ldots$} & $\ldots$ & $\ldots$ \\
$b=4$ & $(0,2.8)$ & $(0, .8)$ & $(-.66,-.53)$ & $(-1.71,-1.48)$ & $(-3,-2.2)$ & $\ldots$ \\
$b=3$ & $(0,4.6)$ & $(.5,2.1)$ & $(0, .6)$ & $(-1,-.4)$ & $(-2.28,1.11)$ & $\ldots$ \\
$b=2$ & $(0,6.4)$ & $(\mathbf{1 . 3 3}, \mathbf{3 . 0 6})$ & $(\mathbf{1}, \mathbf{1 . 4})$ & $(0, .4)$ & $(-1.33,-.26)$ & $\ldots$ \\
$b=1$ & $(0,8.2)$ & $(\mathbf{3}, 3.2)$ & $(\mathbf{2 . 6 6}, \mathbf{1 . 5 3})$ & $(1.5, .7)$ & $(0, .2)$ & $\ldots$ \\
$b=0$ & $(5,5)$ & $(8,0)$ & $(6,0)$ & $(4,0)$ & $(2,0)$ & $\ldots$ \\
\hline & $a=0$ & $a=1$ & $a=2$ & $a=3$ & $a=4$ & $\ldots$ \\
\hline
\end{tabular}

Note: Payoffs of the restricted game are in bold.

figure. In the discrete game, the parties may be unable to play these equilibrium strategies as they will typically be outside the parties' discrete choice sets. Our proposed procedure implies restricting attention to the four candidate equilibrium - the four points (the vertices of the square) around the continuous equilibrium.

To illustrate, consider a simple game in which the prize at stake is $V=10$, the parties' marginal costs of effort are $c_{\mathrm{A}}=2$ and $c_{\mathrm{B}}=1.8$ and $\varepsilon=1$, so that in the discrete game parties can only choose integer levels of effort. The equilibrium of the continuous game is $a^{*}=$ $\left(1.8 /(2+1.8)^{2}\right) 10=1.24$ and $b^{*}=\left(2 /(2+1.8)^{2}\right) 10=1.38$. The procedure introduced earlier allows us to restrict attention to a subset of the parties' choices, namely, to the integers above and below the continuous equilibrium: $a \in\{1,2\}$ and $b \in\{1,2\}$, as indicated by the four points around $\left(a^{*}, b^{*}\right)$ in figure 1 . Table 1 reports the corresponding parties' payoffs, $(A, B)$, in the discrete game; the payoffs of the restricted game are in bold. It is easy to see that the equilibrium of the restricted game is $\left(a^{*}=1, b^{*}=1\right)$-and that this is also the equilibrium of the full game. (In table 1 , we only report a subset of the game's payoffs, but note that the payoffs become negative as we move further away from the continuous equilibrium; the full table is in the appendix.)

\section{ANALYSIS}

In this section, we investigate the conditions under which the procedure highlighted earlier is valid. To preview our results: proposition 1 shows that if the parties' marginal costs of effort are not "too" asymmetric, the equilibrium of the discrete game is contained in the square cell around the equilibrium of the continuous game and hence the procedure 
introduced earlier can be applied. If this sufficient condition is not met, our procedure may fail to identify the correct equilibrium. Proposition 2 provides the conditions for this equilibrium to be in pure strategies or in mixed strategies and, in the former case, identifies which of the four vertices of the cell is an equilibrium. Proposition 3 shows that the pure strategy equilibrium (when it exists) is unique and proposition 4 characterizes the mixing probabilities of the mixed strategy equilibria. Proposition 5 and 6 examine when the continuous game is a good approximation of the discrete game and, finally, proposition 7 provides a counterexample, showing that outside the domain of the sufficient conditions given in proposition 1, the solution to the restricted game may not identify the equilibrium of the full game.

To start, let

$$
\begin{aligned}
& \hat{c}_{\mathrm{A}} \equiv \frac{a^{*}-b^{*}}{2 \underline{b^{*}}} \\
& \hat{c}_{\mathrm{B}} \equiv \frac{b^{*}-a^{*}}{2 \underline{a^{*}}}
\end{aligned}
$$

be threshold levels for the parties' marginal costs of effort, $c_{\mathrm{A}}$ and $c_{\mathrm{B}}$. Note that, as $a^{*}$ and $b^{*}$ depend on the costs, these thresholds are only implicitly defined in terms of the equilibrium of the continuous game and they are both equal to zero if the parties' costs of effort are symmetric. In addition, let

$$
\mathbf{E} \equiv\left\{\left(\underline{a^{*}}, \underline{b^{*}}\right),\left(\overline{a^{*}}, \underline{b^{*}}\right),\left(\underline{a^{*}}, \overline{b^{*}}\right),\left(\overline{a^{*}}, \overline{b^{*}}\right)\right\}
$$

be the equilibrium set. This set contains the floor and the ceiling of the equilibrium of the continuous game-that is, the four points around the continuous equilibrium, as depicted in figure 1, and describes the parties' choice sets in the restricted game.

In the next proposition, we will show that, if the parties' marginal costs of effort lie above the thresholds in equation (4) - that is, when $c_{\mathrm{A}} \geq \hat{c}_{\mathrm{A}}$ and $c_{\mathrm{B}} \geq \hat{c}_{\mathrm{B}}$, then solving the restricted game provides a solution for the full game and the equilibrium of the full game is in $\mathbf{E}$.

Most of our results depend on the asymmetry between the parties' marginal costs of effort; figure 2 visualizes the region in which these conditions are satisfied in the $\left(c_{\mathrm{A}}, c_{\mathrm{B}}\right)$ plane for $\varepsilon=1$. Note that the conditions are always satisfied if the parties are symmetric $c_{\mathrm{A}}=c_{\mathrm{B}}$ and that they are more easily satisfied if the prize at stake is large. Note also that if $c_{\mathrm{A}}>c_{\mathrm{B}}$, then we have $a^{*}<b^{*}$ and the condition $c_{\mathrm{A}} \geq \hat{c}_{\mathrm{A}}$ is always satisfied and hence only the threshold $\hat{c}_{\mathrm{B}}$ is binding. Vice versa if $c_{\mathrm{A}}<c_{\mathrm{B}}$. Hence, looking at figure 2, the condition $c_{\mathrm{A}}>\hat{c}_{\mathrm{A}}$ 


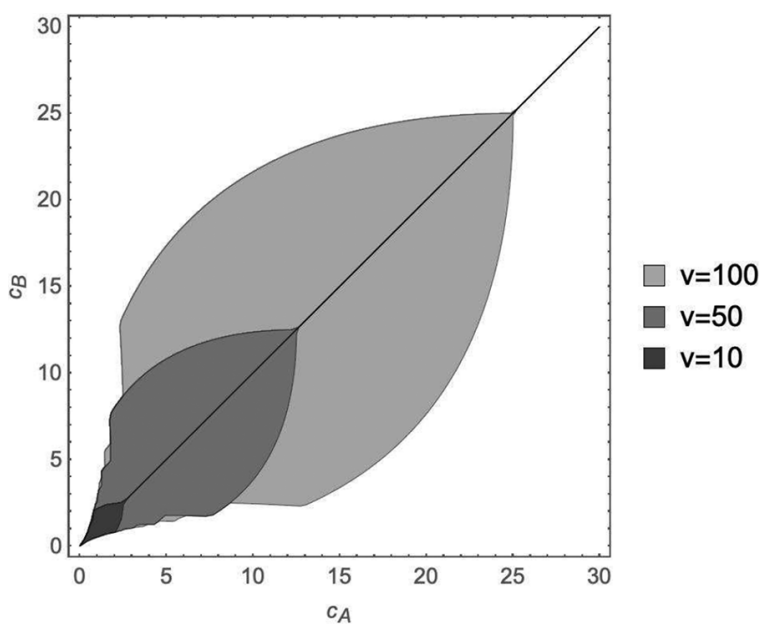

Figure 2. Values of $c_{\mathrm{A}}$ and $c_{\mathrm{B}}$ such that $c_{\mathrm{A}} \geq \hat{c}_{\mathrm{A}}$ and $c_{\mathrm{B}} \geq \hat{c}_{\mathrm{B}}$ (for $\varepsilon=1$ ).

is active above the diagonal, whereas the condition $C_{B}>\hat{C}_{B}$ is active below the diagonal.

Proposition 1. If $c_{\mathrm{A}} \geq \hat{c}_{\mathrm{A}}$ and $c_{\mathrm{B}} \geq \hat{c}_{\mathrm{B}}$, the discrete game has a Nash equilibrium that lies in the equilibrium set $\mathbf{E}$.

\section{Proof of Proposition 1. See appendix. QED}

Proposition 1 shows that if the parties are not too asymmetric concerning their marginal costs of effort, the discrete game has an easyto-find solution that lies in the smallest (discrete) neighborhood around the equilibrium of the continuous game. Then we can apply an easy transformation rule, which we provide in the next proposition. The intuition for the result in proposition 1 is straightforward. The number of choices available to a party in the discrete game depends on that party's cost of effort. A large cost of effort implies that a party has few effort levels to choose from. Think of a game with $V=10, \varepsilon=1$, and $c_{\mathrm{A}}=5$ : party A can effectively choose to exert effort $a=0$ (thereby spending 0 ), effort $a=1$ (which costs 5 ), or effort $a=2$ (which costs as much as the prize, 10). Imagine that party B's cost of effort is $c_{\mathrm{B}}=1$. Repeating the same reasoning, it is easy to see that the effective choices available to party $\mathrm{B}$ are $b \in\{0,1, \ldots .10\}$, so that party $B$ can more easily fine-tune his or her effort level around what would be theoretically optimal if party B could choose among a continuum of options. In contrast, party A's choices are coarser. This asymmetry in the parties' ability to optimize their behavior causes our procedure 
to break down because the party with the finer decision set can exploit the other party's coarseness to his or her advantage, as will be further explained when commenting on proposition 7 . Note also that changes in $v$ and in $\varepsilon$ affect the scale of the problem, but do not change the relative size of the parties' costs, which generates the relevant asymmetry.

Under the conditions of proposition 1, we can solve the full game simply by solving the restricted game. In the following proposition, we study the characteristics of the equilibrium of the restricted game: namely, whether the game has a pure or a mixed strategy equilibrium and, if the equilibrium is in pure strategies, which of the four points in $\mathbf{E}$ is the equilibrium point. Let

$$
\begin{aligned}
& \underline{c_{\mathrm{A}}} \equiv \frac{\underline{b^{*}}}{\left(\underline{a^{*}}+\varepsilon+\underline{b^{*}}\right)\left(\underline{a^{*}}+\underline{b^{*}}\right)} V \\
& \underline{c_{\mathrm{B}}} \equiv \frac{\underline{a^{*}}}{\left(\underline{a^{*}}+\varepsilon+\underline{b^{*}}\right)\left(\underline{a^{*}}+\underline{b^{*}}\right)} V \\
& \overline{c_{\mathrm{A}}} \equiv \frac{\underline{b^{*}}+\varepsilon}{\left(\underline{a^{*}}+\underline{b^{*}}+2 \varepsilon\right)\left(\underline{a^{*}}+\underline{b^{*}}+\varepsilon\right)} V \\
& \overline{c_{\mathrm{B}}} \equiv \frac{a^{*}+\varepsilon}{\left(\underline{a^{*}}+\underline{b^{*}}+2 \varepsilon\right)\left(\underline{a^{*}}+\underline{b^{*}}+\varepsilon\right)} V
\end{aligned}
$$

and note that if $c_{\mathrm{A}}=c_{\mathrm{B}}$, the four thresholds above collapse to two identical thresholds that we denote as $\underline{c} \equiv v / 2\left(2 \underline{a}^{*}+\varepsilon\right)=v / 2\left(2 \underline{b^{*}}+\varepsilon\right)$.

Proposition 2. If $c_{\mathrm{A}} \geq \hat{c}_{\mathrm{A}}$ and $c_{\mathrm{B}} \geq \hat{c}_{\mathrm{B}}$, then the equilibrium of the discrete game is a pure strategy Nash or a mixed strategy Nash equilibrium as indicated in tables 2-4.

Proof of Proposition 2. See appendix. QED

The next figures visualize the results of proposition 2. Figure 3 describes the characteristics of the equilibrium as a function of the parties' marginal costs of effort within the conditions set in proposition 1 .

Table 2. Equilibria If $c_{\mathrm{A}}<c_{\mathrm{B}}$

\begin{tabular}{l|ccc}
\hline$c_{\mathrm{B}}>\underline{c_{\mathrm{B}}}$ & $\left(\overline{a^{*}}, \underline{b^{*}}\right)$ & $\left(\underline{a^{*}}, \underline{b^{*}}\right)$ & $\left(\underline{a^{*}}, \underline{b^{*}}\right)$ \\
$\overline{c_{\mathrm{B}}} \leq c_{\mathrm{B}} \leq \underline{c_{\mathrm{B}}}$ & $\overline{\left(\overline{a^{*}}, \underline{b^{*}}\right)}$ & Mixed & $\left(\underline{a^{*}}, \overline{b^{*}}\right)$ \\
$c_{\mathrm{B}}<\overline{c_{\mathrm{B}}}$ & $\left(\overline{a^{*}}, \overline{b^{*}}\right)$ & $\left(\overline{a^{*}}, \overline{b^{*}}\right)$ & $\left(\underline{a^{*}}, \overline{b^{*}}\right)$ \\
\hline & $c_{\mathrm{A}} \leq \underline{c_{\mathrm{A}}}$ & $\underline{c_{\mathrm{A}}}<c_{\mathrm{A}}<\overline{c_{\mathrm{A}}}$ & $c_{\mathrm{A}} \geq \overline{c_{\mathrm{A}}}$ \\
\hline
\end{tabular}


Table 3. Equilibria If $c_{\mathrm{A}}=c_{\mathrm{B}}$

\begin{tabular}{l|c}
\hline$\left.\overline{a^{*}}, \overline{b^{*}}\right)$ & $\left(\underline{a^{*}}, \underline{b^{*}}\right)$ \\
\hline$c \leq \underline{c}$ & $c>\underline{c}$ \\
\hline
\end{tabular}

Table 4. Equilibria If $c_{\mathrm{A}}>c_{\mathrm{B}}$

\begin{tabular}{l|lll}
\hline$c_{\mathrm{B}} \geq \overline{c_{\mathrm{B}}}$ & $\left.\overline{\left(a^{*}\right.}, \underline{b^{*}}\right)$ & $\left(\overline{a^{*}}, \underline{b^{*}}\right)$ & $\left(\underline{a^{*}}, \underline{b^{*}}\right)$ \\
$\underline{c_{\mathrm{B}}}<c_{\mathrm{B}}<\overline{c_{\mathrm{B}}}$ & $\left.\overline{\left(a^{*}\right.}, \overline{b^{*}}\right)$ & Mixed & $\left(\underline{a^{*}}, \underline{b^{*}}\right)$ \\
$c_{\mathrm{B}} \leq \underline{c_{\mathrm{B}}}$ & $\left(\overline{a^{*}}, \overline{b^{*}}\right)$ & $\left(\underline{a^{*}}, \overline{b^{*}}\right)$ & $\left(\underline{a^{*}}, \overline{b^{*}}\right)$ \\
\hline & $c_{\mathrm{A}}<\overline{c_{\mathrm{A}}}$ & $\overline{c_{\mathrm{A}}} \leq c_{\mathrm{A}} \leq \underline{c_{\mathrm{A}}}$ & $c_{\mathrm{A}}>\underline{c_{\mathrm{A}}}$ \\
\hline
\end{tabular}

Next, we show how the position of the continuous game within the cell with vertices in $\mathbf{E}$ affects the characteristics of the corresponding equilibrium of the discrete game. Figure 4 depicts the characteristics of the discrete equilibrium in the plane with coordinates $\left(a^{*}, b^{*}\right)$ for $V=100$ and $\varepsilon=1$. The graph shows how the position of the continuous equilibrium $\left(a^{*}, b^{*}\right)$ within the square cell surrounding it affects the type of equilibrium of the discrete game.

For instance, take the square cell that is closer to the origin in figure 4-the graph starts at $(1,1)$ because the conditions in proposition 1 bound the solution away from zero-and notice that if the continuous

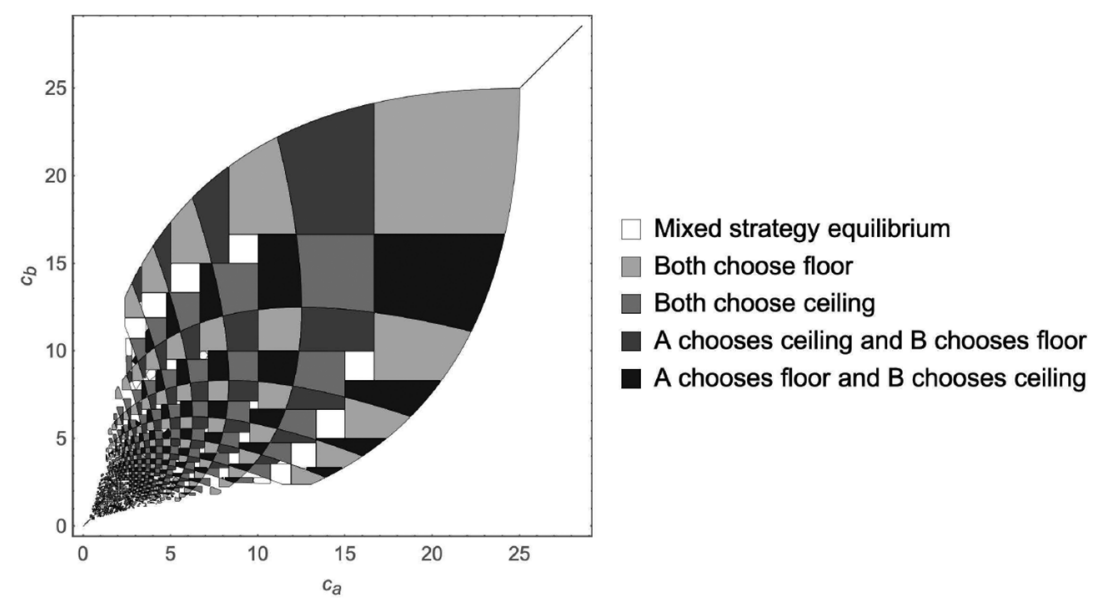

Figure 3. Equilibrium of the discrete game for different costs of effort $(v=100$ and $\varepsilon=1$ ). 


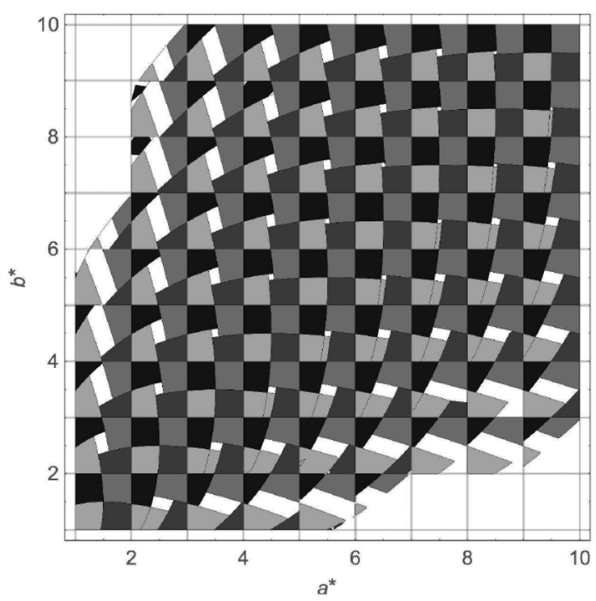

Mixed strategy equilibrium

Both choose floor

Both choose ceiling

A chooses ceiling and $B$ chooses floor

A chooses floor and B chooses ceiling

Figure 4. Equilibrium of the discrete game as a function of the equilibrium of the continuous game $(v=100$ and $\varepsilon=1)$.

equilibrium is close to $(1,1)$, then the discrete game has a symmetric equilibrium at $(1,1)$. Conversely, if the continuous equilibrium is close to $(2,2)$, then the equilibrium of discrete game will be $(2,2)$. Similarly, asymmetric equilibria of the discrete game will emerge when the continuous equilibrium approaches $(1,2)$ or $(2,1)$. In this case, there is no room for mixed strategy equilibria. The next cell to the right, however, allows for such a possibility when the continuous equilibrium $\left(a^{*}, b^{*}\right)$ is somewhat in the middle of the cell.

The following proposition shows that the pure strategy equilibria identified thanks to propositions 1 and 2 are unique.

Proposition 3. If $c_{\mathrm{A}} \geq \hat{c}_{\mathrm{A}}$ and $c_{\mathrm{B}} \geq \hat{c}_{\mathrm{B}}$, and if the discrete game has a pure strategy Nash equilibrium, the equilibrium is unique.

\section{Proof of Proposition 3. See appendix. QED}

Next, we examine the features of the mixed strategy equilibria and, in particular, we characterize the mixing probabilities.

Proposition 4. If $c_{\mathrm{A}} \geq \hat{c}_{\mathrm{A}}$ and $c_{\mathrm{B}} \geq \hat{c}_{\mathrm{B}}$, and if the discrete game has a mixed strategy Nash equilibrium, then the equilibrium mixing probabilities are

$$
\begin{aligned}
& p_{\mathrm{A}}^{*}=\frac{c_{\mathrm{B}}-\overline{c_{\mathrm{B}}}}{\frac{{\underline{c_{\mathrm{B}}}}-\overline{c_{\mathrm{B}}}}{c_{\mathrm{B}}}}(\mathrm{A}) \\
& p_{\mathrm{B}}^{*}=\frac{c_{\mathrm{A}}-\overline{c_{\mathrm{A}}}}{\underline{c_{\mathrm{A}}}-\overline{c_{\mathrm{A}}}}(\mathrm{B})
\end{aligned}
$$


so that party A chooses $\underline{a}^{*}$ with probability $p_{\mathrm{A}}^{*}$ and chooses $\overline{a^{*}}$ with the complementary probability $1-p_{\mathrm{A}}^{*}$. Similarly, party B chooses $\underline{b^{*}}$ with probability $p_{\mathrm{B}}^{*}$ and chooses $b^{*}$ with the complementary probability $1-p_{\mathrm{B}}^{*}$.

Proof of Proposition 4. See appendix. QED

\section{CONTINUOUS APPROXIMATION \\ OF DISCRETE RENT-SEEKING GAMES}

The results in proposition 1 show under what conditions the discrete rent-seeking game can be easily solved by restricting attention to a neighborhood $\mathbf{E}$ of the equilibrium of the continuous game. Here we address the related question of, within these conditions, how good the continuous approximation of a discrete equilibrium is. The following two propositions show that the size of the contested prize, the asymmetry in the parties' costs of effort, and the coarseness of the parties' discrete effort sets affect the approximation in intuitive ways. Finally, proposition 7 shows that if the conditions of proposition 1 are violated, our proposed procedure may fail.

Proposition 5. The conditions $c_{\mathrm{A}} \geq \hat{c}_{\mathrm{A}}$ and $c_{\mathrm{B}} \geq \hat{c}_{\mathrm{B}}$ are more easily satisfied if:

- The party's decision sets are dense ( $\varepsilon$ is small).

- The prize at stake is large ( $v$ is large).

- The parties' marginal costs of effort are symmetric $\left(c_{\mathrm{A}}\right.$ and $c_{\mathrm{B}}$ are close to each other).

Proof of Proposition 5. See appendix. QED

Proposition 6. If $c_{\mathrm{A}} \geq \hat{c}_{\mathrm{A}}$ and $c_{\mathrm{B}} \geq \hat{c}_{\mathrm{B}}$, mixed strategy equilibria are less likely if the parties' marginal costs of effort are symmetric $\left(c_{\mathrm{A}}\right.$ and $c_{\mathrm{B}}$ are close to each other).

Proof of Proposition 6. See appendix. QED

Proposition 7. If $c_{\mathrm{A}}<\hat{c}_{\mathrm{A}}$ or $c_{\mathrm{B}}<\hat{c}_{\mathrm{B}}$, the procedure detailed in proposition 2 may fail to identify the correct equilibrium.

Proof of Proposition 7. See appendix. QED 


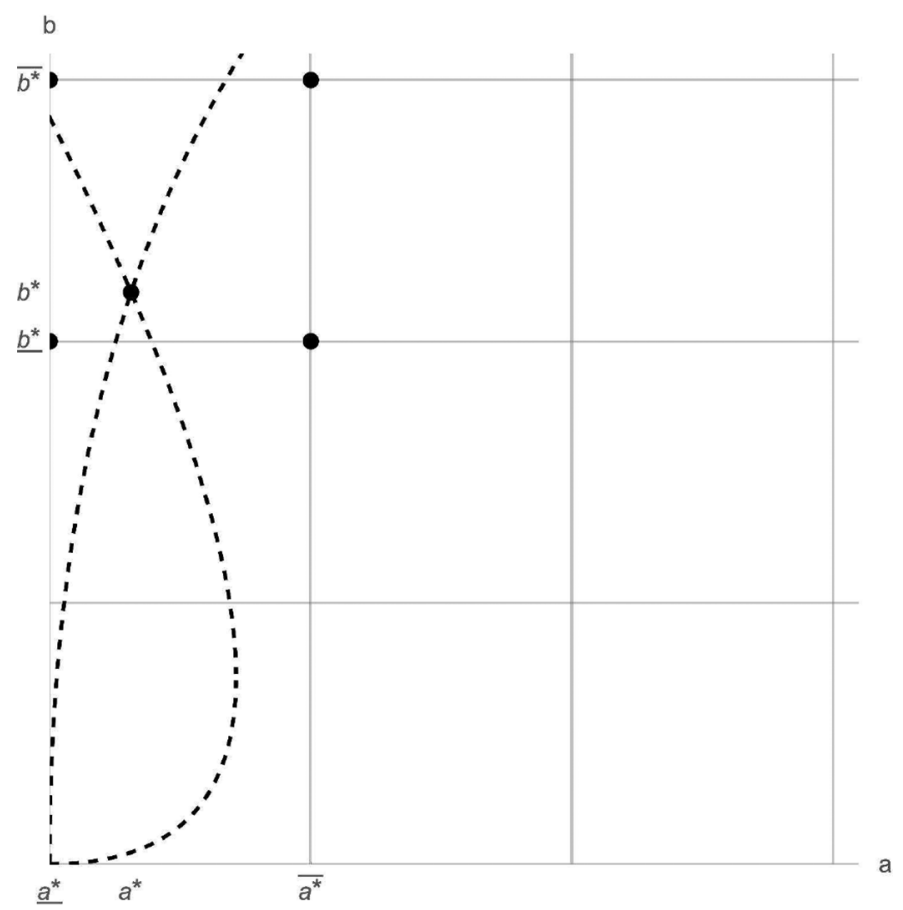

Figure 5. Candidate equilibria of a discrete game with $v=10$, $\mathrm{c}_{\mathrm{A}}=3.5, \mathrm{c}_{\mathrm{B}}=0.5$, and $\varepsilon=1$.

The proof of proposition 7 proceeds by example $\left(v=10, c_{\mathrm{A}}=3.5\right.$, $c_{\mathrm{B}}=0.5$, and $\varepsilon=1$. .). It is easy to verify that $c_{\mathrm{B}}<\hat{c}_{\mathrm{B}}$ and hence proposition 1 does not apply. (Moreover, because $c_{\mathrm{A}} / c_{\mathrm{B}}>3$, we are in region I in fig. A2.) As shown in the appendix, in this case the procedure set out in proposition 2 would select $(0,2)$ as an equilibrium. However, as shown in figure 5 , party $\mathrm{B}$ has an incentive to deviate and, namely, prefers $(0,1)$, which is outside the equilibrium set, over $(0,2)$, because the former is closer to $B^{\prime}$ s reaction function. Hence, $(0,2)$ cannot be an equilibrium of the full game.

\section{SYMMETRIC PARTIES}

Consider now the case in which the parties are perfectly symmetric, that is, $c_{\mathrm{A}}=c_{\mathrm{B}}=c$, the parties' choice sets consist of the nonnegative integers, and the prize at stake is equal to $v=1$. The solution of the corresponding continuous game is notoriously $a^{*}=b^{*}=1 / 4 c$. By proposition 1 , we can focus attention on the restricted game with $a \in\{(1 / 4 c), \overline{(1 / 4 c)}\}$ and $b \in\{(1 / 4 c), \overline{(1 / 4 c)}\}$, that is, the parties' action set includes only the integer above and the one below the 
solution of the continuous game. Let $(1 / 4 c) \equiv y$ and $\overline{(1 / 4 c)}=y+1$. We have the following payoffs for party $\mathrm{A}$ and $\mathrm{B}$, respectively:

\begin{tabular}{c|c|c|}
\multicolumn{1}{c}{$b=y$} & $b=y+1$ \\
\cline { 2 - 3 }$a=y+1$ & $\left(\frac{y+1}{2 y+1}-c(y+1), \frac{y}{2 y+1}-c y\right)$ & $\left(\frac{1}{2}-c(y+1), \frac{1}{2}-c(y+1)\right)$ \\
\cline { 2 - 3 }$a=y$ & $\left(\frac{1}{2}-c y, \frac{1}{2}-c y\right)$ & $\left(\frac{y}{2 y+1}-c y, \frac{y+1}{2 y+1}-c(y+1)\right)$ \\
\cline { 2 - 3 } & &
\end{tabular}

Note that, because the parties are symmetric, then $(a=y, b=y)$ is a Nash equilibrium if neither party finds it advantageous to deviate unilaterally to $y+1$, that is, iff $(1 / 2)-c y>((y+1) /(2 y+1))-c(y+1)$. If this condition is satisfied, there cannot be an asymmetric equilibrium in which one party plays $y$ and the other plays $y+1$. The former condition is equivalent to:

$$
y>\frac{1}{4 c}-\frac{1}{2} .
$$

Similarly, $(a=y+1, b=y+1)$ is a Nash equilibrium if $(1 / 2)-c(y+1)>$ $(y /(2 y+1))-c y$, which again guarantees that there is no asymmetric equilibrium. The former condition is equivalent to

$$
y<\frac{1}{4 c}-\frac{1}{2} .
$$

Because the two conditions (eqq. [5] and [6]) are mutually exclusive, for $y \neq(1 / 4 c)-(1 / 2)$ the game has a unique, symmetric pure strategy Nash equilibrium. (If $y=(1 / 4 c)-(1 / 2)$, both symmetric and asymmetric equilibria are weakly supported. The parties are indifferent between playing $y$ and $y+1$, and there is also a continuum of mixed strategy equilibria supported by any mixing probability.) Condi-

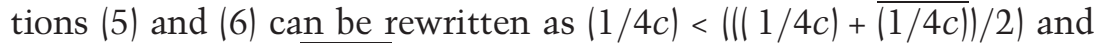
$(1 / 4 c)>(((1 / 4 c)+\overline{(1 / 4 c)}) / 2)$, respectively, where the right-hand side of these inequalities is the midpoint between the integer below and the integer above $1 / 4 c$. So rewritten, these conditions say that, if the equilibrium of the continuous game is below the midpoint, that is, if it is closer to the integer below, both parties will choose the integer below $1 / 4 c$ in the equilibrium of the discrete game; otherwise, they will choose the integer above. This leads to the following result:

Corollary 1. The discrete rent-seeking game with symmetric marginal costs of effort $c, V=1$, and $\varepsilon=1$ has a unique pure strategy Nash equilibrium iff $c \neq 1 /(4 n+2)$ with $n \in\{0,1,2, \ldots\}$; otherwise, the game has four pure strategy Nash equilibria and a 


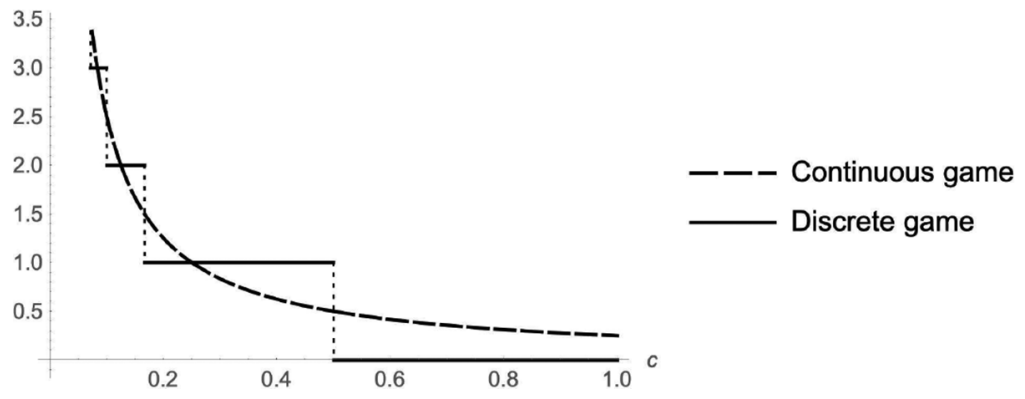

Figure 6. Equilibrium level of effort in the continuous and discrete games.

continuum of mixed strategy Nash equilibria. At the equilibrium, the parties play the integer that is closest to their equilibrium strategy in the continuous game.

The transformation rule from the continuous game to the discrete game is hence very simple: in the discrete game, parties choose the integer that is closest to the equilibrium of the continuous game. Figure 6 illustrates this point. We see that the equilibrium is the integer below if the stepped line is below the curved line and is the integer above otherwise. As $c$ increases, the parties move discontinuously from the integer above to the integer below the equilibrium of the continuous game in a stepwise way. Note that in the symmetric case, mixed strategy equilibria occur only in the (degenerate) case in which the parties are indifferent as to which strategy to play, that is, when the equilibrium of the continuous game is exactly halfway between two admissible discrete levels of effort.

\section{AN APPLICATION TO EVIDENCE PRODUCTION}

\subsection{Setup}

We can now apply the theory developed earlier to evidence production at trial. Experts, documents, and witnesses are typically discrete. Yet reaching out to experts, reviewing documents, and finding and preparing witnesses are costly activities, and one could use the monetary costs thereof as the choice variable. Although money can be thought of as a continuous variable, changing the unit of measurement does not make a discrete strategy space continuous. In the evidence production game, money is an input into the discovery of evidence, not the choice being made.

We consider two parties-a plaintiff $\Pi$ and a defendant $\Delta$-who are in a dispute about a contested amount of money equal to $v$ to be 
shared according to $x \in(0,1)$. On one interpretation, the plaintiff has a contract claim against the defendant with $V x$ as the true amount of damages suffered by the plaintiff. An omniscient court would award $v x$ to plaintiff; but, though the parties know $x$, the court does not. Thus, the court must rely on evidence submitted by the parties.

A piece of evidence is a signal $s \in\{-1,1\}$, where $s=1$ occurs with probability $p(x)$ and is a signal in favor of the plaintiff, whereas $s=-1$ occurs with the complementary probability $1-p(x)$ and is a signal in favor of the defendant, with $p(x)$ monotonically increasing in $x$. This simple structure captures the idea that the plaintiff has a higher probability of finding evidence in his or her favor if he or she has suffered a larger loss, and vice versa for the defendant. In a perfectly symmetric setup, we would have that $1-p(1-x)=p(x)$, which implies $p(1 / 2)=1 / 2$ and first-order symmetry $p_{x}(x)=p_{x}(1-x)$. A simple example of symmetric probabilities of finding evidence is given by $p(x)=x$. We will analyze the general case and comment on the symmetric case when needed.

Each party endogenously decides how many signals to collect. Then, at trial, the court issues a judgment $t=\left(\sigma_{1} /\left(\sigma_{1}+\sigma_{-1}\right)\right)_{V}$ (with $t=1 / 2$ if $\left.\sigma_{1}=\sigma_{-1}=0\right)$, where $\sigma_{1} \equiv \Sigma(s \mid s=1)$ and $\sigma_{-1} \equiv-\Sigma(s \mid s=-1)$. More plainly, $\sigma_{1}$ is the number of signals $s=1$ submitted to the court and, similarly, $\sigma_{-1}$ is the number of signals $s=-1$. The court simply considers the relative number of signals submitted by each party the "weight of the evidence." Note that, if evidence were generated randomly, $t$ would be equal to the number of successes over the number of trials from a Bernoulli distribution with probability $p(x)$ and hence would be an unbiased estimator of $x$ iff $p(x)=x$, which also satisfies first-order symmetry as noted earlier. Evidence, however, is not a random process: parties strategically decide how much to invest in evidence production and, among the pieces of evidence they find, parties decide which to submit to the court and which to withhold. ${ }^{3}$ Before delving into the evidence production process, let us first summarize the timing of the game: ${ }^{4}$

- Time 0: A dispute arises; the parties jointly observe $x$ and the plaintiff files a lawsuit at no cost.

- Time 1: The parties endogenously and simultaneously decide how many signals to collect (at a cost per signal).

- Time 2: Each party decides which of the collected signals to submit to court, and the court adjudicates the case.

\footnotetext{
${ }^{3}$ We thus assume that neither party has an obligation to reveal unfavorable evidence. In criminal trials in the United States, prosecutors do have an obligation to reveal exculpatory evidence.

${ }^{4}$ We abstract from settlement because it is not essential to our analysis.
} 
The court is a mechanical player that adjudicates the case simply based on the evidence submitted according to the rule of decision $t$. Accordingly, because $t$ increases in $\sigma_{1}$ and decreases in $\sigma_{-1}$, each party has incentives to submit all favorable signals and to withhold all signals that are favorable to the other party. Hence, the game unfolds at the evidence production stage.

\subsection{Endogenous Investments in Litigation}

Before trial, at time 2, the parties invest in attorney-hours with the purpose of gathering evidence for trial. Each attorney draws an average of 1 signal per hour at a cost $k_{\Pi}$ and $k_{\Delta}$, respectively. For simplicity, we assume that the expected time needed to collect each extra signal is constant. This allows us to describe evidence collection as a Poisson process; as each party only submits favorable signals, the relevant Poisson process has an arrival rate of favorable signals equal to $p(x)$ for the plaintiff and $1-p(x)$ for the defendant. ${ }^{5}$ This is a possibly restrictive assumption, as it is more likely that collecting an additional signal costs more time than the previous signals; yet it greatly simplifies the analysis.

Evidence collection is a sequential process that stops when the marginal value of spending an additional hour of effort equals its marginal cost. However, recall that a Poisson process is memoryless and, hence, if it makes sense to invest additional time to find a new signal at time $t$, it also makes sense to keep investigating at time $t^{\prime}>t$, indeed to keep investigating until that signal is found, because the additional expected waiting time is constant as we go forward. Crucially, the parties decide when to stop collecting signals independently of each other. Therefore, the question reduces to how many (favorable) signals the parties would collect in the Nash equilibrium of a game with simultaneous moves where the costs of collection are the expected costs from the sequential Poisson process.

To calculate these costs, let us start with the plaintiff. Given that the arrival rate of favorable signals is $p(x)$ signals per hour, the average time needed to collect one signal is $1 / p(x)$ hours and hence the expected time needed to collect $\sigma_{\Pi}$ favorable signals is $\sigma_{\Pi} / p(x)$ hours at an expected cost of $\sigma_{\mathrm{II}} k_{\Pi} / p(x)$. Similarly, the defendant expects to spend $\sigma_{\Delta} k_{\Delta} /(1-p(x))$ to collect $\sigma_{\Delta}$ favorable signals.

The investments in attorney-hours are chosen by the parties to maximize their expected trial outcome net of the costs. The parties'

\footnotetext{
${ }^{5}$ Note that, in a Poisson process with base arrival rate $\lambda$, if only a fraction $p(x)$ of the draws are kept and the rest is discarded, the resulting arrival rate of favorable signals is $p(x) \lambda$. In our model, we have $\lambda=1$ and hence the plaintiff's arrival rate of favorable signals is $p(x)$ whereas the defendant's arrival rate is $1-p(x)$.
} 
decision problems are

$$
\begin{aligned}
& \max _{\sigma_{\Pi}}\left[\frac{\sigma_{\Pi}}{\sigma_{\Pi}+\sigma_{\Delta}} V-\frac{\sigma_{\Pi} k_{\Pi}}{p(x)}\right] \quad \text { (plaintiff) } \\
& \min _{\sigma_{\Delta}}\left[\frac{\sigma_{\Pi}}{\sigma_{\Pi}+\sigma_{\Delta}} V+\frac{\sigma_{\Delta} k_{\Delta}}{1-p(x)}\right] \text { (defendant) }
\end{aligned}
$$

where the plaintiff maximizes the amount of money he or she will collect from the defendant at trial net of his or her evidence collection costs; similarly, the defendant minimizes the amount of money he or she will pay to the plaintiff plus his or her evidence collection costs. Using $\sigma_{\Delta} /\left(\sigma_{\Pi}+\sigma_{\Delta}\right)=1-\left(\sigma_{\Pi} /\left(\sigma_{\Pi}+\sigma_{\Delta}\right)\right)$, we can rewrite the parties' objectives more conveniently as

$$
\begin{aligned}
& \max _{\sigma_{\Pi}}\left[\frac{\sigma_{\Pi}}{\sigma_{\Pi}+\sigma_{\Delta}} V-\frac{\sigma_{\Pi} k_{\Pi}}{p(x)}\right] \quad \text { (plaintiff) } \\
& \max _{\sigma_{\Delta}}\left[\frac{\sigma_{\Delta}}{\sigma_{\Pi}+\sigma_{\Delta}} V-\frac{\sigma_{\Delta} k_{\Delta}}{1-p(x)}-V\right] \text { (defendant) }
\end{aligned}
$$

Assuming for now that $\sigma_{\Pi}$ and $\sigma_{\Delta}$ are continuous variables, the first-order conditions lead to the following result:

$$
\begin{aligned}
& \sigma_{\Pi}^{*}=\frac{p(x)(1-p(x))}{\left(p(x) k_{\Delta}+(1-p(x)) k_{\Pi}\right)^{2}} v p(x) k_{\Delta} \\
& \sigma_{\Delta}^{*}=\frac{p(x)(1-p(x))}{\left(p(x) k_{\Delta}+(1-p(x)) k_{\Pi}\right)^{2}} v(1-p(x)) k_{\Pi}
\end{aligned} .
$$

Note that, in the continuous strategy equilibrium, although the parties collect different amounts of evidence- $-\sigma_{\Pi}^{*} \neq \sigma_{\Delta}^{*}$ unless $p(x)=$ $1-p(x)$ - they spend the same amount of resources in attorney fees:

$$
\frac{\sigma_{\Pi}^{*} k_{\Pi}}{p(x)}=\frac{\sigma_{\Delta}^{*} k_{\Delta}}{1-p(x)}=\frac{p(x)(1-p(x))}{\left(p(x) k_{\Delta}+(1-p(x)) k_{\Pi}\right)^{2}} k_{\Pi} k_{\Delta} V .
$$

This is because the parties play a rent-seeking game with constant marginal returns to effort. Therefore, the total cost of evidence production at trial (the total rent dissipation) is

$$
D=2 \frac{p(x)(1-p(x))}{\left(p(x) k_{\Delta}+(1-p(x)) k_{\Pi}\right)^{2}} k_{\Pi} k_{\Delta} V .
$$

The analysis in section 3 can be applied to this case. We know therefore that if $k_{\Pi} / p(x) \leq\left(\sigma_{\Pi}^{*}-\sigma_{\Delta}^{*}\right) / 2 \sigma_{\Delta}^{*}$ and $k_{\Delta} /(1-p(x)) \leq\left(\sigma_{\Pi}^{*}-\sigma_{\Pi}^{*}\right) /$ $2 \sigma_{I}^{*}$, at the equilibrium of the discrete game the plaintiff will choose 
either $\sigma_{\text {II }}^{*}$ or $\overline{\sigma_{\text {II }}^{*}}$ or a mix of the two and, likewise, the defendant will choose either $\sigma_{\Delta}^{*}$ or $\sigma_{\Delta}^{*}$ or a mix of the two.

\section{CONCLUSIONS AND APPLICATIONS}

We have identified a class of discrete rent-seeking games for which the equilibrium of the associated continuous rent-seeking game serves as an approximation of the equilibrium of the discrete game. This analysis is useful for two reasons. First, identifying the equilibria of the discrete game may be a difficult task as the payoff matrix may be very large. Identifying the equilibrium of the continuous game is relatively straightforward. Our analysis dramatically simplifies the analysis to a simple twostep procedure. One first determines the equilibrium of the continuous game. Then one examines the four discrete points in the mesh closest to the continuous equilibrium. Moreover, we identify factors that make the continuous equilibrium a better approximation of the equilibrium in the discrete game.

Second, discrete rent-seeking games are substantively important as they are the natural way to model widespread social phenomena. Aspects of litigation provide the most obvious example. The incentive effects of legal rules are mediated by the litigation process as people act ex ante in the shadow of the law, that is, in the shadow of the expected outcome at trial. That outcome, of course, depends on the behavior of the litigating parties in the gathering and presentation of evidence. But evidence is not a continuous variable; it comes in discrete bundles. Each litigant must decide which bundles to gather and to submit to the court. Many other phenomena share this discrete structure. Companies, for instance, acquire patents to restrict entry into the industry; again, patents are a discrete quantity.

Finally, we wish to emphasize two limitations of our analysis and suggest possible extensions. First, we assumed that the parties' discrete effort sets are equally spaced, that is, that the parties can choose among similar sets of possibilities. This assumption holds in all of the applications that we suggested. In reality, however, there could be cases in which a party can choose among a set of discrete options that is finer than the set available to the other party. Think, for instance, of a situation in which party A's effort set consists of the nonnegative integers whereas party B's effort set consists of multiples of $1 / 2$ and hence party B has more options to choose from. ${ }^{6}$ We conjecture that our results will qualitatively apply to this case, although the exact conditions will necessarily change quantitatively.

${ }^{6}$ That is, we have $\varepsilon_{\mathrm{A}} \neq \varepsilon_{\mathrm{B}}$. 
The intuition is that a change in the relative coarseness of the parties' choice sets results in an asymmetry that has similar effects on the parties' abilities to optimize their behavior as the parties' costs of effort, as emphasized in the introduction. Second, we have provided an analysis of simultaneous rent-seeking games, whereas in reality parties often choose sequentially. Again, we conjecture that our main results will qualitatively apply to a sequential game, although the specific quantitative aspects of our results will necessarily change.

\section{A P P E N D I X}

\section{A1. Example}

In table A1, we report the parties' payoffs for all feasible levels of effort in relation to the example in table 1 and figure 1.

\section{A2. Proof of Proposition 1}

Proof. To prove the proposition, it is sufficient to show that unilateral deviations outside the equilibrium set $\mathbf{E}$ reduce the payoff of the deviating party. We will focus on party B's decisions under the assumption that party A has chosen either $a^{*}$ or $a^{*}$. (Applying the same logic to party A will then be trivial.) The proof is structured as follows. We will first offer some useful preliminary observations on party B's reaction function, which will allow us to partition the space into three different regions. We will then prove the results of the proposition by analyzing each of these regions in turn.

A2.1. Preliminary Observations. Party B's reaction function, $b^{* *}(a)$, as given in equation (3), is depicted in figure A1.

Note that the slope of $b^{* *}(a)$ is:

1. $\frac{d b^{* *}}{d a}=1$ at $a=\frac{V}{16 c_{\mathrm{B}}}$;

2. $\frac{d b^{* *}}{d a}=0$ at $a=\frac{v}{4 C_{\mathrm{B}}}$;

3. $\frac{d b^{* *}}{d a}>-1$ at $a=\frac{v}{c_{B}}$.

Let us now consider the possible location of the equilibrium of the continuous game $\left(a^{*}, b^{*}\right)$ as defined in equation (2), which must necessarily lie along $\mathrm{B}^{\prime}$ s reaction function. For a given $c_{\mathrm{B}}$, the dashed lines in figure A2 depict party A's reaction function at different levels of $\mathrm{A}^{\prime}$ s marginal cost of effort, $c_{\mathrm{A}}$. The following observations will be useful later. 


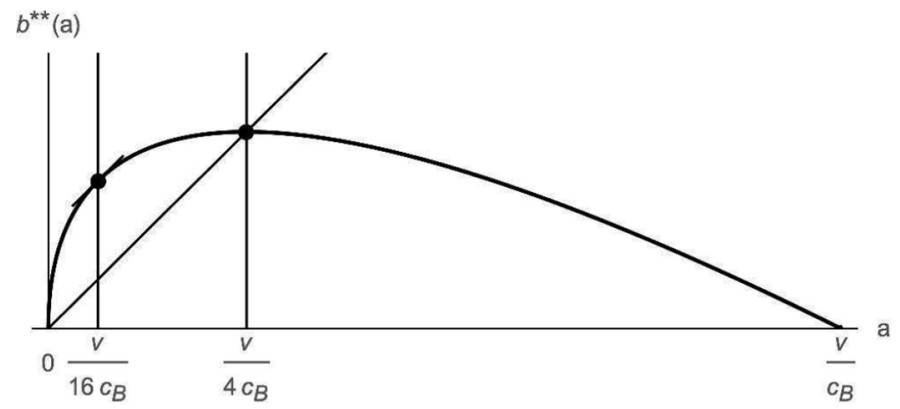

Figure A1. Party B's reaction function.

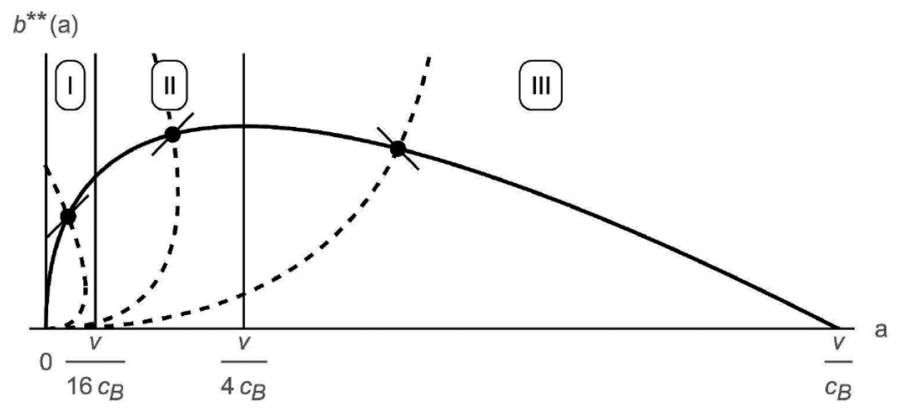

Figure A2. Slope of $b^{* *}(a)$ at different equilibria of the continuous game.

Lemma 1. Using (2) and the slope of $b^{* *}(a)$, we can identify three regions of party $\mathrm{B}^{\prime} \mathrm{s}$ reaction function as depicted in figure $\mathrm{A} 2$ :

Region I: If $c_{\mathrm{A}}>3 c_{\mathrm{B}}$, we have $a^{*}<v / 16 c_{\mathrm{B}}$ and the slope of $b^{* *}(a)$ is $d b^{* *} / d a>1$; therefore, in region I, the line with slope 1 and that passes through the equilibrium of the continuous game $\left(a^{*}, b^{*}\right)$ crosses $b^{* *}(a)$ from above.

Region II: If $c_{\mathrm{B}} \leq c_{\mathrm{A}} \leq 3 c_{\mathrm{B}}$, we have $v / 16 c_{\mathrm{B}} \leq a^{*} \leq v / 4 c_{\mathrm{B}}$ and the slope of $b^{* *}(a)$ is $0 \leq\left(d b^{* *} / d a\right) \leq 1$; therefore, in region II, the line with slope 1 and that passes through the equilibrium of the continuous game $\left(a^{*}, b^{*}\right)$ crosses $b^{* *}(a)$ from below.

Region III: If $c_{\mathrm{A}}<c_{\mathrm{B}}$, we have $v / 4 c_{\mathrm{B}}<a^{*} \leq v / c_{\mathrm{B}}$ and the slope of $b^{* *}(a)$ is $-1<\left(d b^{* *} / d a\right)<0$; in region III, the line with slope -1 that passes through the equilibrium of the continuous game $\left(a^{*}, b^{*}\right)$ crosses $b^{* *}(a)$ from above.

Proof of Lemma 1. Omitted. QED 
In the discrete game, because each party can choose among a countable set of (equally spaced) points, the possible equilibria of the game can be visualized as the nodes of a grid in the $(a, b)$ space. The line with slope 1 or -1 that passes through the equilibrium of the continuous game will be important because it limits the number of points that we will need to consider in the proof, as will be clear later.

A2.2. Region III. We can now start tackling the proof by beginning from region III. Further, let us start by assuming that party A has chosen $a^{*}$ and then repeat the exercise for $a^{*}$.

A2.2.1 Region III: Party A Chooses $\overline{a^{*}}$. Party B's possible effort choices can be visualized as the points $\left(a^{*}, b\right)$ with $b \in\{0, \varepsilon, 2 \varepsilon, \ldots\}$, which are located along the vertical line passing through $\frac{a^{*}}{a^{*}}$; see figure A3. We will show that party B's payoff at any point $\left(a^{*}, b\right)$ with $b>\overline{b^{*}}$ is less than at the point $\left(a^{*}, \overline{b^{*}}\right)$ and, similarly, that $\mathrm{B}^{\prime}$ s payoff at any point $\left(\overline{a^{*}}, b\right)$ with $b<\underline{b^{*}}$ is less than at the point $\left(\overline{a^{*}}, \underline{b^{*}}\right)$. That is: party $\mathrm{B}$ does not have an incentive to deviate unilaterally outside the set $\mathbf{E}$, given party A's choice of $\overline{a^{*}}$. (Repeating the exercise for the case of $a=a^{*}$ will conclude the proof for region III. We will then run the same analysis in regions II and I.)

To start with, note that, although the point $\left(\overline{a^{*}}, \overline{b^{*}}\right)$ is always above $\mathrm{B}^{\prime}$ s reaction function, the point $\left(\overline{a^{*}}, \underline{b^{*}}\right)$ could be either below or above B's reaction function. This is due to the fact that $\mathrm{B}^{\prime} \mathrm{s}$ reaction function-which passes through $\left(a^{*}, b^{*}\right)$-decreases in $a$ and hence is below the horizontal $b^{*}$-line when $a>a^{*}$. This in turn implies that all the points $\left(\overline{a^{*}}, b\right)$ above the horizontal $b^{*}$-line also lie above B's reaction function - as is the case for the point $\left(\overline{a^{*}}, \overline{b^{*}}\right)$ - whereas the points $\left(a^{*}, b\right)$ below the horizontal $b^{*}$-line may lie above or below $\mathrm{B}^{\prime}$ s reaction function. Therefore, the point $\left(\overline{a^{*}}, \underline{b^{*}}\right)$ could be either below or above $\mathrm{B}^{\prime} \mathrm{s}$ reaction function. Let us consider these two cases in turn.

If $\left(\overline{a^{*}}, \underline{b^{*}}\right)$ is below $\mathrm{B}^{\prime}$ s reaction function, we are in the case depicted in figure $\mathrm{A} 3$, where the points $\left(\overline{a^{*}}, b\right)$ with $\underline{b}<\underline{b^{*}}$ lie below $\mathrm{B}^{\prime}$ s reaction function and the points $\left(a^{*}, b\right)$ with $b>b^{*}$ lie above $\mathrm{B}^{\prime}$ s reaction function. Given that B's payoff is concave in $b$ and maximal along B's reaction function, it follows that B's payoff decreases if B moves above $\overline{b^{*}}$ or below $\underline{b^{*}}$, which proves that B will not deviate outside the equilibrium set $\mathbf{E}$.

If instead $\left(\overline{a^{*}}, \underline{b^{*}}\right)$ is above $\mathrm{B}^{\prime}$ s reaction function, as depicted in figure A4, the reasoning above breaks down because now we need to compare $\left(\overline{a^{*}}, b^{*}\right)$-which, by hypothesis, is above $\mathrm{B}^{\prime}$ s reaction function-with points $\left(\overline{a^{*}}, b\right)$ with $b<\underline{b^{*}}$ possibly below B's reaction function, and concavity does not help us solve this problem. The point $\left.\overline{\left(a^{*}\right.}, \overline{b^{*}}\right)$ still dominates all points $\left(\overline{a^{*}}, b\right)$ above it, but we need 
to show that $\left(\overline{a^{*}}, \underline{b^{*}}\right)$ dominates all points $\left(\overline{a^{*}}, b\right)$ below it. To do so, we will first establish an intermediate result.

Let

$$
\hat{b}^{-}(a)=a^{*}+b^{*}-a
$$

be the line with slope -1 that passes through the equilibrium of the continuous game (the dotted diagonal in figure A4), which we mentioned in Lemma 1 above; then:

Lemma 2. Given party A's choice of effort, $a$, party B's payoff is weakly greater at $b=b^{*}$ than at $b=\hat{b}^{-}(a)$.

Proof of Lemma 2. We need to show that $\left(b^{*} /\left(a+b^{*}\right)\right) v-$ $b^{*} c_{\mathrm{B}} \geq\left(\hat{b}^{-}(a) /\left(a+\hat{b}^{-}(a)\right) \|_{V}-\hat{b}^{-}(a) c_{\mathrm{B}}\right.$. Replacing $\hat{b}^{-}(a)$ and rearranging, we have that the latter inequality is satisfied iff

$$
\left(a^{*}-a\right)\left(a v-\left(a^{*}+b^{*}\right)\left(a+b^{*}\right) c_{\mathrm{B}}\right) \leq 0 .
$$

The first factor is positive if $a<a^{*}$ and negative if $a>a^{*}$. Using equation (2), the second factor can be rewritten as

$$
\begin{gathered}
\frac{a\left(c_{\mathrm{A}}+c_{\mathrm{B}}\right)^{3} V-\left(a\left(c_{\mathrm{A}}+c_{\mathrm{B}}\right)^{2}+c_{\mathrm{A}} V\right) c_{\mathrm{B}}}{\left(c_{\mathrm{A}}+c_{\mathrm{B}}\right)^{3}} \\
=\left(a\left(c_{\mathrm{A}}+c_{\mathrm{B}}\right)^{2}-c_{\mathrm{B}} V\right) \frac{c_{\mathrm{A}}}{\left(c_{\mathrm{A}}+c_{\mathrm{B}}\right)^{3}} V,
\end{gathered}
$$

which is positive iff $a>\left(c_{\mathrm{B}} /\left(c_{\mathrm{A}}+c_{\mathrm{B}}\right)^{2}\right) V=a^{*}$ and negative if $a<a^{*}$. It follows that the two factors in equation (8) have the opposite sign and hence that the inequality is strictly satisfied if $a<a^{*}$ or $a>a^{*}$. If $a=a^{*}$, then equation (8) holds as an equality. QED

Lemma 2 shows that given $\mathrm{A}^{\prime}$ s choice of $\overline{a^{*}}, \mathrm{~B}^{\prime}$ s payoff is higher when $\mathrm{B}$ chooses the continuous-equilibrium level of effort $b^{*}$ rather than the level of effort $\hat{b}^{-}\left(\overline{a^{*}}\right)$ lying on the line with slope -1 that passes through the continuous equilibrium. Note that, importantly, the lemma compares all possible levels of effort within these sets, thus not only those admitted in the discrete game. In turn, this is not a problem for our purposes because we will use these comparisons of effort levels merely as intermediate steps of the proof. We will now combine lemma 2 with the observations in lemma 1 to prove our result.

First, it is easy to verify graphically that, in the discrete game, the first feasible effort level below $\underline{b^{*}}$ must be at $b \leq \hat{b}^{-}\left(\overline{a^{*}}\right)$, that is, under the line with slope -1 . To see why, note the widest distance between $\underline{b^{*}}$ and $\hat{b}^{-}\left(\overline{a^{*}}\right)$ is achieved when, given $\underline{b^{*}}, \hat{b}^{-}\left(\overline{a^{*}}\right)$ is as low as possible, 
that is, when the point $\left(a^{*}, b^{*}\right)$ overlaps with the point $\left(a^{*}, b^{*}\right)$. Yet in this case the first point below $\underline{b}^{*}$ must lie exactly on the line with slope -1 , which proves that the observation above is correct.

From lemma 1 we know that the line $\hat{b}^{-}(a)$ crosses $\mathrm{B}^{\prime}$ s reaction function from above. Looking at figure A4, notice that, if A plays $a^{*}$, $\mathrm{B}^{\prime}$ s payoff is greater at $\hat{b}^{-}\left(\overline{a^{*}}\right)$ than at any point below it because of the concavity of B's reaction function. By lemma 2, B's payoff is greater at $b^{*}$ than at $\hat{b}^{-}\left(\overline{a^{*}}\right)$. Further, $\mathrm{B}^{\prime}$ s payoff is greater at $b^{*}$ than it is at $b^{*}$ again due to concavity, because both points are above $\mathrm{B}^{\prime}$ s reaction function but $\overline{b^{*}}$ is closer to it. By transitivity, $\mathrm{B}^{\prime}$ s payoff is greater at $\overline{b^{*}}$ than at any point below even when $\bar{b}^{*}$ is above $B^{\prime}$ s reaction function. (Note again that it is immaterial whether $b^{*}$ and $\hat{b}^{-}\left(\overline{a^{*}}\right)$ are in party $\mathrm{B}^{\prime}$ s discrete choice set because they are never actually chosen.)

A2.2.2 Region III: Party A Chooses $a^{*}$. We can now prove that if A plays $a^{*}$, then B's payoff is greater at $b^{*}$ than at any point above it. Similarly to what we noted earlier, the point $\left(a^{*}, \underline{b}^{*}\right)$ is always below $\mathrm{B}^{\prime}$ s reaction function, but the point $\left(a^{*}, \overline{b^{*}}\right)$ could be above or below it. Yet because lemma 2 applies equally to the left and to the right of $a^{*}$, the proof is a trivial replication of the reasoning above. Combining the previous results, we can conclude that in region III party B does not have an incentive to deviate outside the equilibrium set $\mathbf{E}$.

A2.3. Region II. Let us now turn to region II. Let $\hat{b}^{+}(a)=a+b^{*}-a^{*}$ be the line with slope 1 that passes through the equilibrium of the continuous game as shown in figure A5, then:

Lemma 3. In region II, given party A's choice of effort, a, party $\mathrm{B}^{\prime}$ s payoff is weakly greater at $b=b^{*}$ than at $b=\hat{b}^{+}(a)$ iff $c_{\mathrm{B}} \geq$ $\left(b^{*}-a^{*}\right) / 2 a^{*}$.

Proof of Lemma 3. We need to show that, under the conditions set out in the lemma, $\left(b^{*} /\left(a+b^{*}\right) \mid v-b^{*} c_{\mathrm{B}} \geq\left(\hat{b}^{+}(a) /\right.\right.$ $\left(a+\hat{b}^{+}(a) \|_{V}-\hat{b}^{+}(a) c_{\mathrm{B}}\right.$. Replacing $\hat{b}^{+}(a), a^{*}$, and $b^{*}$, and rearranging, we have that the latter inequality is satisfied iff

$$
\frac{\left(c_{\mathrm{A}}+c_{\mathrm{B}}\right)^{2}-c_{\mathrm{B}} V^{2}\left(2 a\left(c_{\mathrm{A}}+c_{\mathrm{B}}\right)^{2} c_{\mathrm{B}}-\left(c_{\mathrm{A}}-c_{\mathrm{B}}\right) v\right)}{\left(c_{\mathrm{A}}+c_{\mathrm{B}}\right)^{2}\left(a\left(c_{\mathrm{A}}+c_{\mathrm{B}}\right)^{2}+c_{\mathrm{A}} V\right)\left(2 a\left(c_{\mathrm{A}}+c_{\mathrm{B}}\right)^{2}+\left(\left(c_{\mathrm{A}}-c_{\mathrm{B}}\right) v\right)\right.} \geq 0 .
$$

Given that in region II we have $c_{\mathrm{A}}>c_{\mathrm{B}}$, the denominator is positive. The numerator is weakly positive iff $a \geq\left(\left(c_{\mathrm{A}}-c_{\mathrm{B}}\right) /\left(2\left(c_{\mathrm{A}}+c_{\mathrm{B}}\right)^{2} c_{\mathrm{B}}\right)\right) V$. Given that we are interested in cases in which party A plays $a \geq \underline{a^{*}}$, the condition becomes 


$$
\underline{a^{*}} \geq \frac{c_{\mathrm{A}}-c_{\mathrm{B}}}{2\left(c_{\mathrm{A}}+c_{\mathrm{B}}\right)^{2} c_{\mathrm{B}}} V=\frac{b^{*}-a^{*}}{2 c_{\mathrm{B}}}
$$

or

$$
c_{\mathrm{B}} \geq \frac{b^{*}-a^{*}}{2 \underline{a^{*}}} .
$$

QED

Lemma 3 provides a result that is analogous to lemma 2, with the only relevant difference that the lemma is now applicable only to the right of a specified threshold level of $a$. As is clear from figure A5, also in this case, two of the relevant points in the set $\mathbf{E}$ can be either above or below B's reaction function, and we can use lemma 3 to prove the result as we did with lemma 2 . We can therefore apply the reasoning developed for region III to region II to show that party B does not have incentives to deviate outside the equilibrium set $\mathbf{E}$ whenever the condition in lemma 3 is satisfied.

A2.4. Region I. Note that the condition in equation (11) can never be satisfied in region I. Hence, this proposition applies to region III and a portion of region II but does not apply to region I.

Repeating the same exercise for party A's reaction function and combining the results completes the proof. QED

\section{A3. Proof of Proposition 2}

Proof. Note that under the conditions of proposition 1 the point $(0,0)$ is not part of the equilibrium set $\mathbf{E}$ unless $c_{\mathrm{A}}=c_{\mathrm{B}}$, a case that we discuss in section 5 . Hence, here we can be sure that the discrete game is bounded away from zero, that is, $\underline{a}^{*} \geq 1$ and $\underline{b^{*}} \geq 1$. Given the results in proposition 1 , we can focus on the restricted game with $a \in\left\{\underline{a^{*}}, a^{*}\right\}$ and $b \in\left\{\underline{b^{*}}, \overline{b^{*}}\right\}$. Note that $a^{*}=\underline{a^{*}}+\varepsilon$ and $\overline{b^{*}}=\underline{b^{*}}+\varepsilon$. We have the

\begin{tabular}{|c|c|c|}
\hline & $b=\underline{b^{*}}$ & $b=\underline{b^{*}}+\varepsilon$ \\
\hline$a=\underline{a^{*}}+\varepsilon$ & $\begin{array}{c}\left(\frac{\frac{a^{*}}{a^{*}}+\varepsilon}{+\varepsilon+\underline{b^{*}}} V-c_{\mathrm{A}}\left(\underline{a^{*}}+\varepsilon\right),\right. \\
\left.\frac{b^{*}}{\underline{a^{*}}+\varepsilon+\underline{b^{*}}} V-c_{\mathrm{B}} \underline{b^{*}}\right)\end{array}$ & 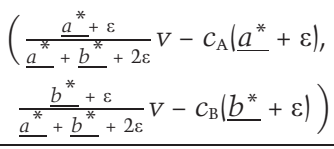 \\
\hline$a=\underline{a^{*}}$ & $\begin{array}{l}\left(\frac{a^{*}}{a^{*}+b^{*}} V-c_{\mathrm{A}} \underline{a^{*}}\right. \\
\left.\frac{b^{*}}{\underline{a}^{*}+\underline{b^{*}}} V-c_{\mathrm{B}} \underline{b^{*}}\right)\end{array}$ & $\begin{array}{c}\left(\frac{\underline{a^{*}}}{\underline{a^{*}}+\varepsilon+\underline{b^{*}}} V-c_{\mathrm{A}} \underline{a^{*}},\right. \\
\left.\frac{\underline{b^{*}}+\varepsilon}{\underline{a^{*}}+\varepsilon+\underline{b^{*}}} V-c_{\mathrm{B}}\left(\underline{b^{*}}+\varepsilon\right)\right)\end{array}$ \\
\hline
\end{tabular}
following payoffs matrix for $(A, B)$ : 
The equilibrium conditions are

- $\left(a=\underline{a^{*}}, b=\underline{b^{*}}\right)$ is a Nash equilibrium if

$$
\begin{aligned}
& \frac{\underline{a^{*}}}{\underline{a^{*}}+\underline{b^{*}}} V-c_{\mathrm{A}} \underline{a^{*}}>\frac{\underline{a^{*}}+\varepsilon}{\underline{a^{*}}+\varepsilon+\underline{b^{*}}} V-c_{\mathrm{A}}\left(\underline{a^{*}}+\varepsilon\right) \\
& \frac{\underline{b^{*}}}{\underline{a}^{*}+\underline{b^{*}}} V-c_{\mathrm{B}} \underline{b^{*}}>\frac{\underline{b^{*}}+\varepsilon}{\underline{a^{*}}+\varepsilon+\underline{b^{*}}} V-c_{\mathrm{B}}\left(\underline{b^{*}}+\varepsilon\right)
\end{aligned}
$$

or

$$
\begin{gathered}
c_{\mathrm{A}}>\frac{\underline{b^{*}}}{\left(\underline{a^{*}}+\varepsilon+\underline{b^{*}}\right)\left(\underline{a^{*}}+\underline{b^{*}}\right)} V \equiv \underline{c_{\mathrm{A}}}(\mathrm{A}) \\
c_{\mathrm{B}}>\frac{\underline{a^{*}}}{\left(\underline{a^{*}}+\varepsilon+\underline{b^{*}}\right)\left(\underline{a^{*}}+\underline{b^{*}}\right)} V \equiv \underline{c_{\mathrm{B}}}(\mathrm{B})
\end{gathered}
$$

- $\left(a=\underline{a}^{*}+\varepsilon, b=\underline{b^{*}}+\varepsilon\right)$ is a Nash equilibrium if

$$
\begin{aligned}
& \frac{a^{*}+\varepsilon}{\underline{a}^{*}+\underline{b^{*}}+2 \varepsilon} V-c_{\mathrm{A}}\left(\underline{a^{*}}+\varepsilon\right)>\frac{\underline{a^{*}}}{\underline{a}^{*}+\varepsilon+\underline{b^{*}}} V-c_{\mathrm{A}} \underline{a^{*}} \\
& \frac{\underline{b}^{*}+\varepsilon}{\underline{a}^{*}+\underline{b^{*}}+2 \varepsilon} V-c_{\mathrm{B}}\left(\underline{b^{*}}+\varepsilon\right)>\frac{\underline{b^{*}}}{\underline{a^{*}}+\varepsilon+\underline{b^{*}}} V-c_{\mathrm{B}} \underline{b^{*}}
\end{aligned}
$$

or

$$
\begin{aligned}
& c_{\mathrm{A}}<\frac{\underline{b^{*}}+\varepsilon}{\left(\underline{a^{*}}+\underline{b^{*}}+2 \varepsilon\right)\left(\underline{a^{*}}+\underline{b^{*}}+\varepsilon\right)} V \equiv \overline{c_{\mathrm{A}}}(\mathrm{A}) \\
& c_{\mathrm{B}}<\frac{a^{*}+\varepsilon}{\left(\underline{a^{*}}+\underline{b^{*}}+2 \varepsilon\right)\left(\underline{a^{*}}+\underline{b^{*}}+\varepsilon\right)} V \equiv \overline{c_{\mathrm{B}}}(\mathrm{B})
\end{aligned}
$$

- $\left(a=\underline{a^{*}}+\varepsilon, b=\underline{b^{*}}\right)$ is a Nash equilibrium if

$$
\begin{aligned}
& c_{\mathrm{A}}<\underline{\underline{c_{\mathrm{A}}}}(\mathrm{A}) \\
& c_{\mathrm{B}}>\overline{c_{\mathrm{B}}}(\mathrm{B})
\end{aligned}
$$

- $\left(a=\underline{a^{*}}, b=\underline{b^{*}}+\varepsilon\right)$ is a Nash equilibrium if

$$
\begin{aligned}
& c_{\mathrm{A}}>\overline{c_{\mathrm{A}}}(\mathrm{A}) \\
& c_{\mathrm{B}}<\underline{c_{\mathrm{B}}}(\mathrm{B})
\end{aligned}
$$

- There is a mixed strategy equilibrium in all other cases. 
Note that if $\underline{a}^{*}>\underline{b^{*}}$, we have $\overline{c_{\mathrm{A}}}>\underline{c_{\mathrm{A}}}$ (and hence $c_{\mathrm{A}}<\underline{c_{\mathrm{A}}}$ and $c_{\mathrm{A}}>\overline{c_{\mathrm{A}}}$ cannot be simultaneously satisfied) and $\overline{c_{B}}>\overline{c_{B}}$ (and hence $c_{B}>\underline{c_{B}}$ and $c_{\mathrm{B}}<\overline{\mathrm{C}_{\mathrm{B}}}$ cannot be simultaneously satisfied) because:

$$
\begin{aligned}
& \overline{c_{\mathrm{A}}} \geq \underline{c_{\mathrm{A}}} \\
& \Leftrightarrow \\
& \frac{\underline{b^{*}}+\varepsilon}{\left(\underline{a^{*}}+\underline{b^{*}}+2 \varepsilon\right)\left(\underline{a^{*}}+\underline{b^{*}}+\varepsilon\right)} \geq \frac{\underline{b^{*}}}{\left(\underline{a^{*}}+\varepsilon+\underline{b^{*}}\right)\left(\underline{a^{*}}+\underline{b^{*}}\right)} \\
& \Leftrightarrow \\
& \frac{\underline{b^{*}}+\varepsilon}{\underline{a^{*}}+\underline{b^{*}}+2 \varepsilon} \geq \frac{\underline{b^{*}}}{\underline{a^{*}}+\underline{b^{*}}} \\
& \Leftrightarrow \\
& \left(\underline{b^{*}}+\varepsilon\right)\left(\underline{a^{*}}+\underline{b^{*}}\right) \geq \underline{b^{*}}\left(\underline{a^{*}}+\underline{b^{*}}+2 \varepsilon\right) \\
& \Leftrightarrow \\
& \underline{a^{*}}+\underline{b^{*}} \geq 2 \underline{b^{*}} \\
& \Leftrightarrow \\
& \underline{a^{*}} \geq \underline{b^{*}} \\
& \Leftrightarrow \\
& \text { Floor }\left[\frac{c_{\mathrm{B}}}{\left(c_{\mathrm{A}}+c_{\mathrm{B}}\right)^{2}} V\right] \geq \text { Floor }\left[\frac{c_{\mathrm{A}}}{\left(c_{\mathrm{A}}+c_{\mathrm{B}}\right)^{2}} V\right] \\
& \Leftrightarrow \\
& c_{\mathrm{B}} \geq c_{\mathrm{A}}
\end{aligned}
$$

and similarly for the inequality $\underline{c_{B}}>\overline{c_{B}}$. Analogously, if $\underline{a^{*}}<\underline{b^{*}}$, we have $\overline{c_{\mathrm{A}}}<\underline{c_{\mathrm{A}}}$ (and hence $c_{\mathrm{A}}<\underline{c_{\mathrm{A}}}$ and $c_{\mathrm{A}}>\overline{c_{\mathrm{A}}}$ cannot be simultaneously satisfied) and $\overline{c_{\mathrm{B}}}>c_{\mathrm{B}}$ (and hence $c_{\mathrm{B}}>\bar{c}_{\mathrm{B}}$ and $c_{\mathrm{B}}<\overline{C_{\mathrm{B}}}$ cannot be simultaneously satisfied). Let us now verify that the conditions for pure strategy Nash equilibria do not overlap and that there are cases in which the equilibrium is in mixed strategy. Tables 2 and 4 do the work. The proof of the case with $c_{\mathrm{A}}=c_{\mathrm{B}}$ is trivial. QED

\section{A4. Proof of Proposition 3}

Sketch of the Proof. (The full proof is available upon request.) We already know from proposition 1 that points lying on the four lines $a=\underline{a}^{*}, a=\overline{a^{*}}, b=\bar{b}^{*}$, and $b=\underline{b^{*}}$ are dominated by points in $\mathbf{E}$. Hence, to prove the uniqueness of the pure strategy Nash equilibrium, we only 
need to show that every other point on the vertices of the grid in the $(a, b)$ space admits a unilateral deviation to a point on one of these four lines. We will do so by considering the case with $c_{\mathrm{A}} \geq c_{\mathrm{B}}$ (the opposite case is analogous).

Let us start by using the lines $a=a^{*}$ and $b=b^{*}$ to divide the plane into four regions as shown in figure A6 and examine each of them in turn.

Region $1\left(\boldsymbol{a}<\boldsymbol{a}^{*}\right.$ and $\left.\boldsymbol{b} \geq \boldsymbol{b}^{*}\right)$ : Every point in region 1 is above $B^{\prime} \mathrm{s}$ reaction function. Hence, $B$ will find it convenient to deviate from any $b>\overline{b^{*}}$ to $b=\overline{b^{*}}$.

Region $2\left(\boldsymbol{a} \geq \boldsymbol{a}^{*}\right.$ and $\left.\boldsymbol{b} \geq \boldsymbol{b}^{*}\right)$ : Similarly, region 2 is entirely to the right of $A^{\prime}$ s reaction function; $A$ will find it convenient to deviate from any $a>\overline{a^{*}}$ to $a=\overline{a^{*}}$.

Region $3\left(\boldsymbol{a} \geq \boldsymbol{a}^{*}\right.$ and $\left.\boldsymbol{b}<\boldsymbol{b}^{*}\right)$ : Consider again the line $\hat{b}^{-}(a)$ with slope -1 passing through the point $\left\{a^{*}, b^{*}\right\}$, which was introduced in lemma 2 . We can then apply a similar reasoning as we did in proposition 1 to show that for every point below this line, $B$ has an incentive to deviate to $\underline{b}^{*}$, and for every point above this line, $A$ has an incentive to deviate to $a^{*}$.

Region $4\left(\boldsymbol{a}<\boldsymbol{a}^{*}\right.$ and $\left.\boldsymbol{b}<\boldsymbol{b}^{*}\right)$ : Consider the horizontal line $b=\hat{b}$, with $\hat{b}$ such that $a^{* *}(\hat{b})=a^{*}$; this line crosses the point at which $A^{\prime}$ s reaction function intersects the line $a=a^{*}$ for the first time (the second time is obviously for $b=b^{*}$ ), as depicted in figure A6. Note that, by construction, the area above this horizontal line is entirely to the left of $A^{\prime}$ s reaction function and hence $A$ will find it convenient to deviate from any $a<a^{*}$ in this area to $a=a^{*}$. Consider now the area below the horizontal line and draw the line $b=a$, which divides the area into two subareas, as in figure A6. Note that $A$ 's payoff is greater at $a=a^{*}$ than it is at $a=b$ (proof omitted); then apply the same reasoning as for region 3 to show that every point above the diagonal admits a unilateral deviation by $A$ to $a^{*}$. Similarly, $B^{\prime}$ s payoff is greater at $b=b^{*}$ than it is at $b=a$ (proof omitted), and again by the same reasoning, every point below the diagonal admits a deviation to $b^{*}$. This concludes the proof for region 4 .

Summing up, all points in each of the regions in which we have subdivided the plane admit a deviation to a point on one of the four lines passing through $\mathbf{E}$, and we know from proposition 1 that all points on these lines admit a unilateral deviation to a point in E. We can conclude that if there is a pure strategy Nash equilibrium in $\mathbf{E}$, then this equilibrium must be unique. QED 


\section{A5. Proof of Proposition 4}

Proof. In a mixed strategy, A chooses $a^{*}$ with probability $p_{\mathrm{A}}$ and $a^{*}+\varepsilon$ with the complementary probability $1-p_{\mathrm{A}}$, which are such that $\mathrm{B}$ is indifferent between $\underline{b^{*}}$ and $\underline{b^{*}}+\varepsilon$. Similarly, B chooses $\underline{b^{*}}$ with probability $p_{\mathrm{B}}$. We have

$$
\begin{aligned}
& p_{\mathrm{B}}\left[\frac{\underline{a^{*}}}{a^{*}+\underline{b^{*}}} V-c_{\mathrm{A}} \underline{a^{*}}\right]+\left(1-p_{\mathrm{B}}\right)\left[\frac{\underline{a^{*}}}{a^{*}+\varepsilon+\underline{b^{*}}} V-c_{\mathrm{A}} \underline{a^{*}}\right] \\
= & p_{\mathrm{B}}\left[\frac{\underline{a^{*}}+\varepsilon}{\underline{a}^{*}+\varepsilon+\underline{b^{*}}} V-c_{\mathrm{A}}\left(\underline{a^{*}}+\varepsilon\right)\right]+\left(1-p_{\mathrm{B}}\right)\left[\frac{\underline{a^{*}}+\varepsilon}{a^{*}+\underline{b}^{*}+2 \varepsilon} V-c_{\mathrm{A}}\left(\underline{a^{*}}+\varepsilon\right)\right] \\
& p_{\mathrm{A}}\left[\frac{\underline{b^{*}}}{\underline{a}^{*}+\underline{b^{*}}} V-c_{\mathrm{B}} \underline{b^{*}}\right]+\left(1-p_{\mathrm{A}}\right)\left[\frac{\underline{b^{*}}}{a^{*}+\varepsilon+\underline{b^{*}}} V-c_{\mathrm{B}} \underline{b^{*}}\right] \\
= & p_{\mathrm{A}}\left[\frac{\underline{b^{*}}+\varepsilon}{\underline{a}^{*}+\varepsilon+\underline{b^{*}}} V-c_{\mathrm{B}}\left(\underline{b^{*}}+\varepsilon\right)\right]+\left(1-p_{\mathrm{A}}\right)\left[\frac{b^{*}+\varepsilon}{a^{*}+\underline{b^{*}}+2 \varepsilon} V-c_{\mathrm{B}}\left(\underline{b^{*}}+\varepsilon\right)\right]
\end{aligned}
$$

or

$$
\begin{aligned}
& c_{\mathrm{A}}=p_{\mathrm{B}} \underline{c_{\mathrm{A}}}+\left(1-p_{\mathrm{B}}\right) \overline{c_{\mathrm{A}}}(\mathrm{A}) \\
& c_{\mathrm{B}}=p_{\mathrm{A}} \underline{c_{\mathrm{B}}}+\left(1-p_{\mathrm{A}}\right) \overline{c_{\mathrm{B}}}(\mathrm{B})
\end{aligned}
$$

or

$$
\begin{aligned}
p_{\mathrm{A}}^{*} & =\frac{c_{\mathrm{B}}-\overline{c_{\mathrm{B}}}}{\underline{c_{\mathrm{B}}}-\overline{c_{\mathrm{B}}}} \\
p_{\mathrm{B}}^{*} & =\frac{c_{\mathrm{A}}-\overline{c_{\mathrm{A}}}}{\underline{c_{\mathrm{A}}}-\overline{c_{\mathrm{A}}}}
\end{aligned}
$$

QED

\section{A6. Proof of Proposition 5}

Proof. Given that, by definition, we have $\underline{a^{*}} \geq a^{*}-\varepsilon$, the inequality in equation (10) implies the following sufficient condition $a^{*}-\varepsilon \geq$ $\left(\left(c_{\mathrm{A}}-c_{\mathrm{B}}\right) /\left(2\left(c_{\mathrm{A}}+c_{\mathrm{B}}\right)^{2} c_{\mathrm{B}}\right)\right) v$, which can be rewritten as $\varepsilon \leq\left(\left(2 c_{\mathrm{B}}^{2}-c_{\mathrm{A}}+\right.\right.$ $\left.c_{\mathrm{B}}\right) /\left(2\left(c_{\mathrm{A}}+c_{\mathrm{B}}\right)^{2} c_{\mathrm{B}}\right) \mid v$. Repeating the exercise for the case in which $c_{\mathrm{A}}>c_{\mathrm{B}}$, we have the following threshold:

$$
\hat{\varepsilon} \equiv \min \left\{\frac{2 c_{\mathrm{A}}^{2}-c_{\mathrm{B}}+c_{\mathrm{A}}}{2\left(c_{\mathrm{A}}+c_{\mathrm{B}}\right)^{2} c_{\mathrm{A}}} V, \frac{2 c_{\mathrm{B}}^{2}-c_{\mathrm{A}}+c_{\mathrm{B}}}{2\left(c_{\mathrm{A}}+c_{\mathrm{B}}\right)^{2} c_{\mathrm{B}}} V\right\} .
$$

If $\varepsilon \leq \hat{\varepsilon}$, then also the condition in equation (10) must be satisfied. It is easy to see that the condition in equation (12) is more easily satisfied if $v$ is large, if $\varepsilon$ is small, and if the costs are similar. Figure A7 
shows how this sufficient condition relates to the conditions in equation (4) and how sensitive is it to variation in $\varepsilon$. QED

\section{A7. Proof of Proposition 6}

Proof. Let us start from the case in which $c_{\mathrm{A}}<c_{\mathrm{B}}$ (the opposite case is analogous). From proposition 2 , we know that a mix strategy equilibrium occurs in the space $\left[\underline{c_{\mathrm{A}}}, \overline{c_{\mathrm{A}}}\right] \times\left[\overline{\mathrm{c}_{\mathrm{B}}}, \underline{c_{\mathrm{B}}}\right]$. If this space shrinks, the mixed strategy equilibrium becomes less likely. Let us then consider:

$$
\begin{aligned}
\overline{c_{\mathrm{A}}}-\underline{c_{\mathrm{A}}} & =\frac{\underline{b^{*}}+\varepsilon}{\left(\underline{a^{*}}+\underline{b^{*}}+2 \varepsilon\right)\left(\underline{a^{*}}+\underline{b^{*}}+\varepsilon\right)} V-\frac{\underline{b^{*}}}{\left(\underline{a^{*}}+\varepsilon+\underline{b^{*}}\right)\left(\underline{a^{*}}+\underline{b^{*}}\right)} V \\
& =\left[\frac{\underline{a^{*}}-\underline{b^{*}}}{\left(\underline{a^{*}}+\underline{b^{*}}\right)\left(\underline{a^{*}}+\underline{b^{*}}+2 \varepsilon\right)}\right] \frac{\varepsilon V}{\left(\underline{\left.a^{*}+\varepsilon+\underline{b^{*}}\right)}\right.}
\end{aligned}
$$

which decreases to zero if $\underline{a}^{*}-\underline{b^{*}}$ approach zero, which is in turn the case when costs are symmetric. An analogous reasoning applies to the length of the second dimension, $\underline{c_{\mathrm{B}}}-\overline{\mathrm{C}_{\mathrm{B}}}$. QED

\section{A8. Proof of Proposition 7}

Proof. To prove the proposition, it is sufficient to find parameter values such that the algorithm presented in proposition 2 fails to identify the equilibrium. Set $V=10, c_{\mathrm{A}}=3.5, c_{\mathrm{B}}=0.5$, and $\varepsilon=1$. Note that these parameters do not satisfy the conditions in proposition 1 . Then $a^{*}=0.31$ so that $\underline{a}^{*}=0$ and $\overline{a^{*}}=1$; similarly, $b^{*}=2.18$ so that $\underline{b^{*}}=2$ and $\overline{b^{*}}=3$. Hence the equilibrium set is $\mathbf{E}=\{(0,2),(1,2)$, $(0,3),(1,3)\}$. Finally, we have $\underline{c_{\mathrm{A}}}=10 / 3>5 / 2=\overline{c_{\mathrm{A}}}$ and $\underline{c_{\mathrm{B}}}=0<5 / 6=$ $\overline{c_{\mathrm{B}}}$.

Because $c_{\mathrm{A}}>c_{\mathrm{B}}$, we can use table 4 . Because $c_{\mathrm{A}}>\underline{c_{\mathrm{A}}}$ and $\underline{c_{\mathrm{B}}}<$ $c_{\mathrm{B}}<\overline{C_{\mathrm{B}}}$, table 4 suggests that the game should have the symmetric Nash equilibrium $(0,2)$. However, it is easy to verify in table A2 that $(0,2)$ is not an equilibrium because party $\mathrm{B}$ has an incentive to deviate to $(0,1)$. QED 


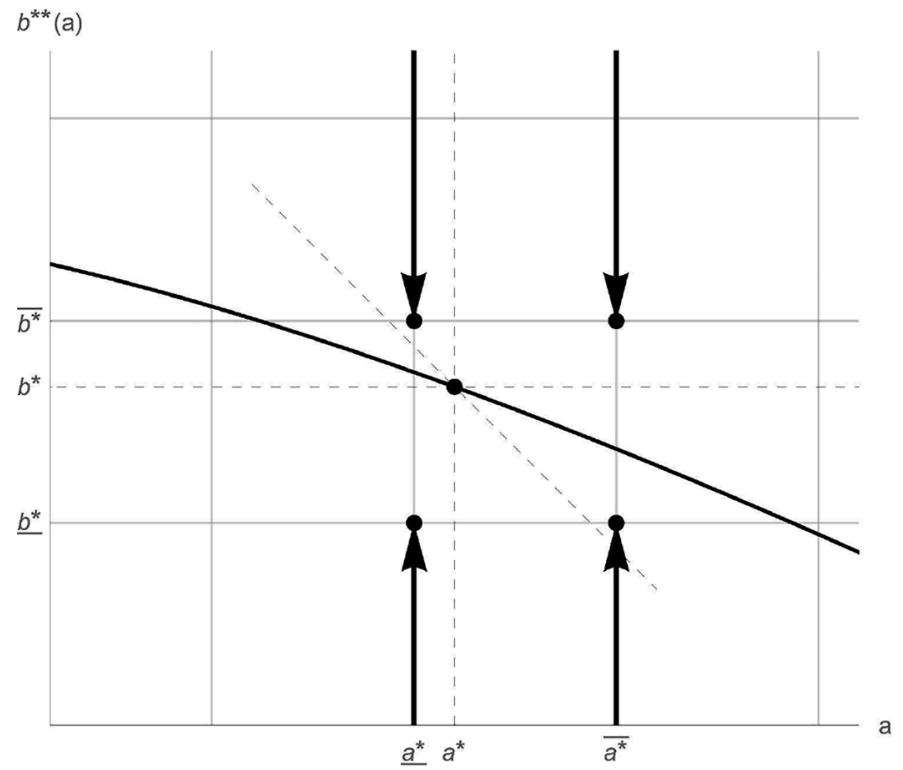

Figure A3. Party B's reactions in region III when $\left(\overline{a^{*}}, \overline{b^{*}}\right)$ is below and $\left(\overline{a^{*}}, b^{*}\right)$ is above $B^{\prime}$ s reaction function.

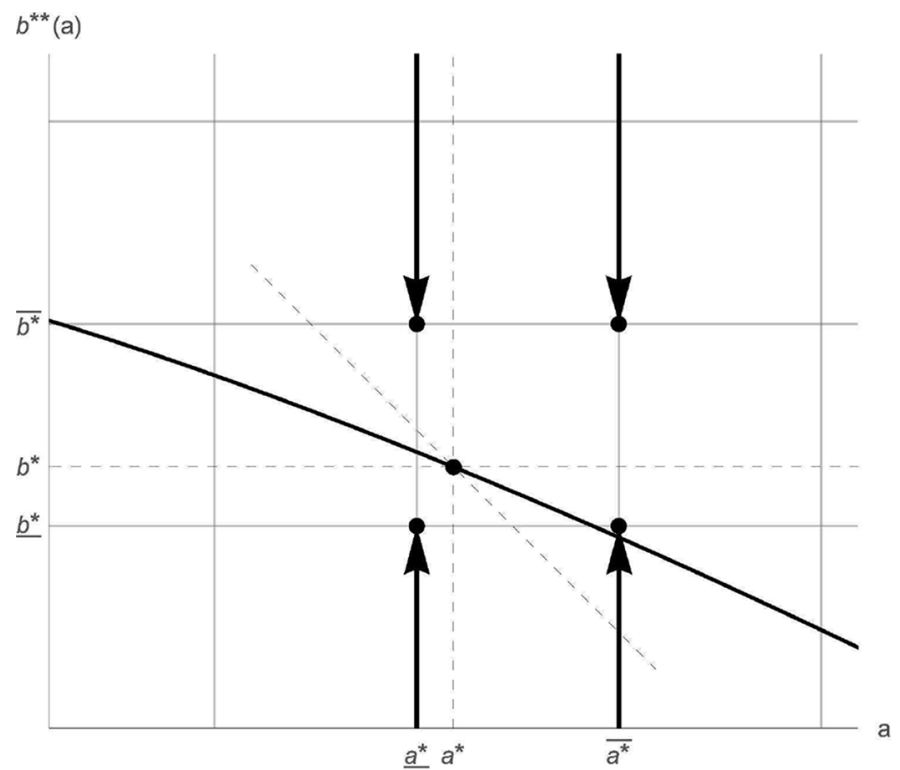

Figure A4. Party B's reactions in region III when both $\left(\overline{a^{*}}, \overline{b^{*}}\right)$ and $\left(\overline{a^{*}}, \underline{b}^{*}\right)$ are above the reaction function. 


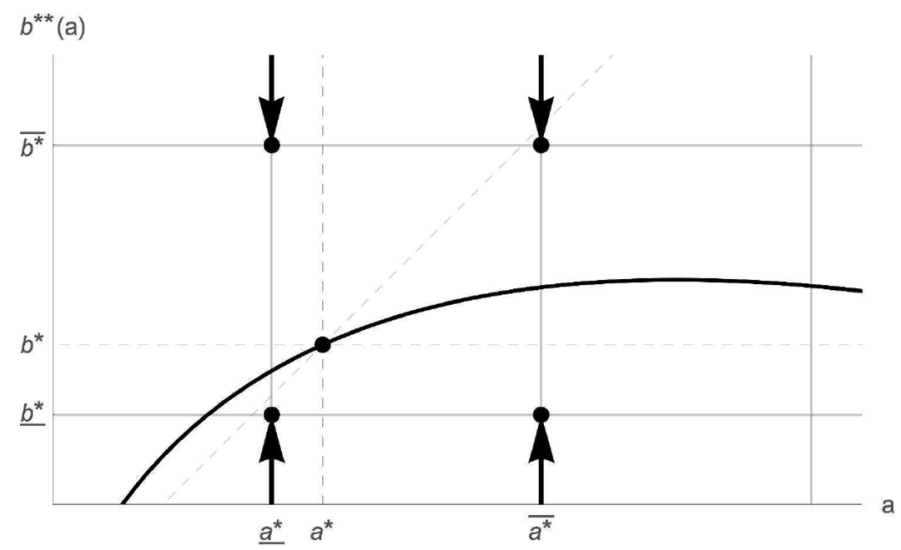

Figure A5. Party B's reactions in region II when $\left(\underline{a}^{*}, \overline{b^{*}}\right)$ is above and $\left(\underline{a}^{*}, \underline{b^{*}}\right)$ is below $B^{\prime}$ s reaction function.

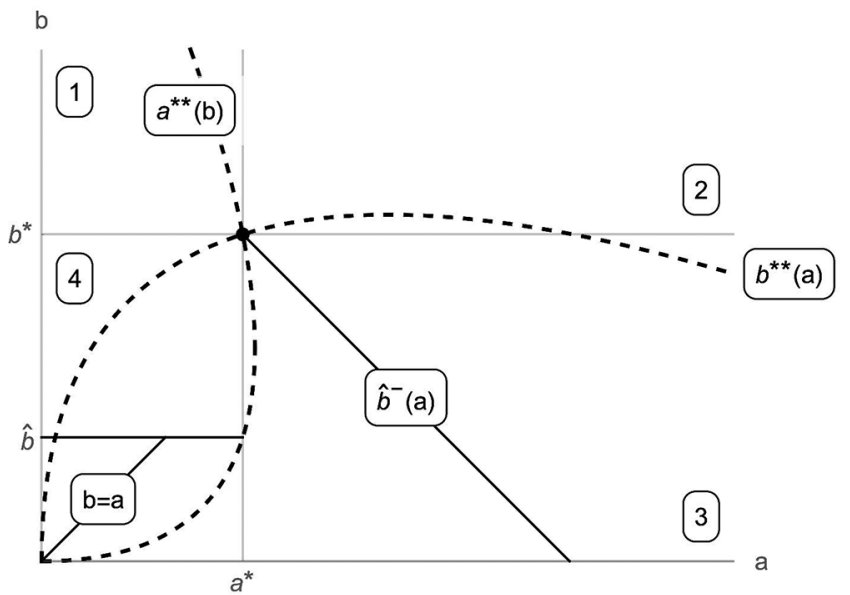

Figure A6. Uniqueness of the pure strategy equilibrium: (a) party B's reaction function; (b) both parties' reaction functions. 


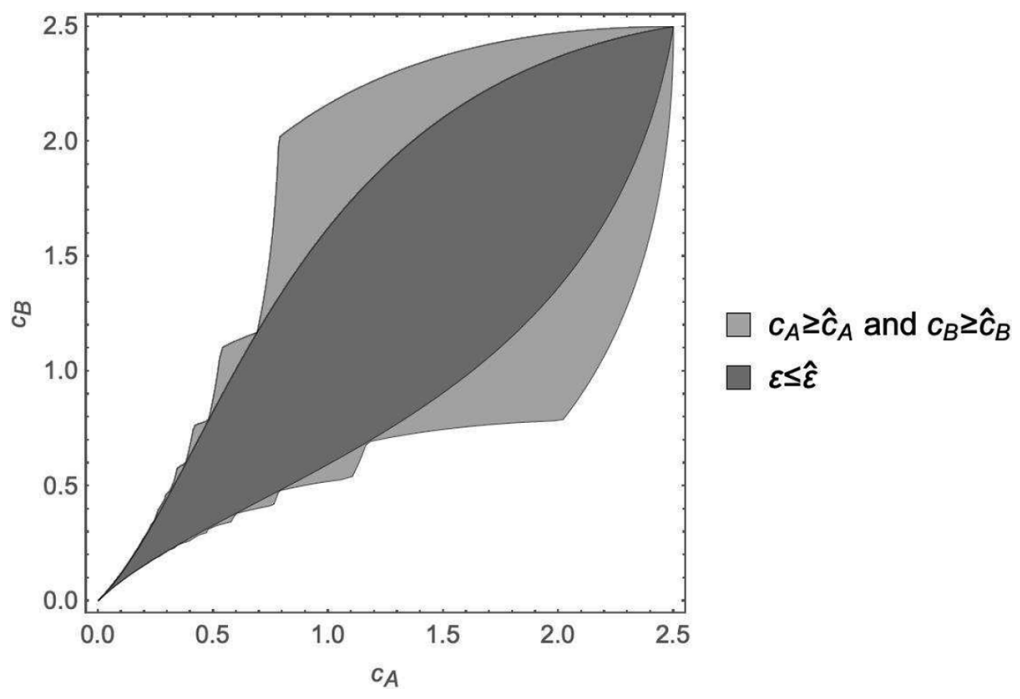

Figure A7. Scope of the sufficient condition in equation $(12)(v=10$ and $\varepsilon=1)$.

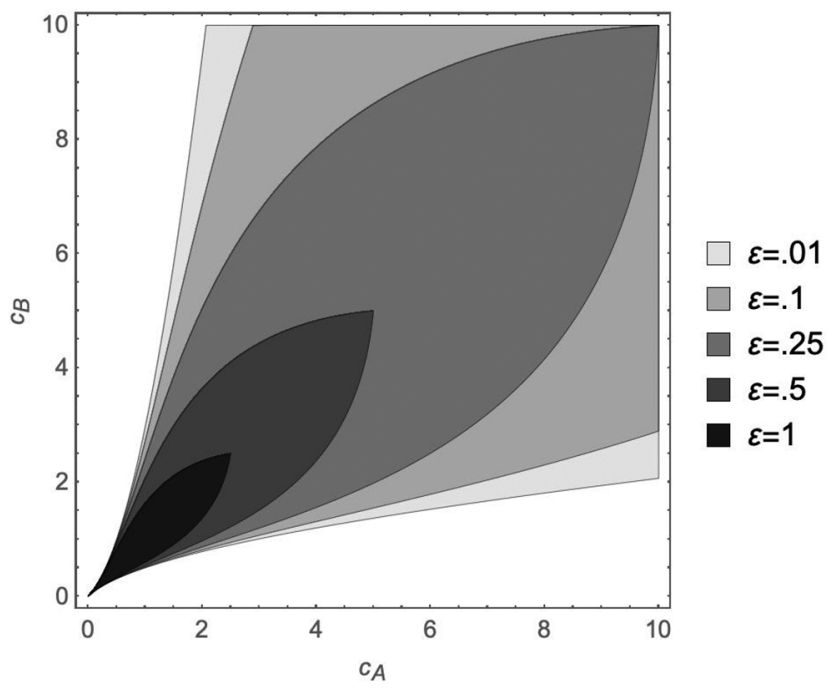

Figure A8. Values of $c_{A}$ and $c_{B}$ for which the sufficient condition in equation (12) is verified for different values of $\varepsilon(v=10)$. 


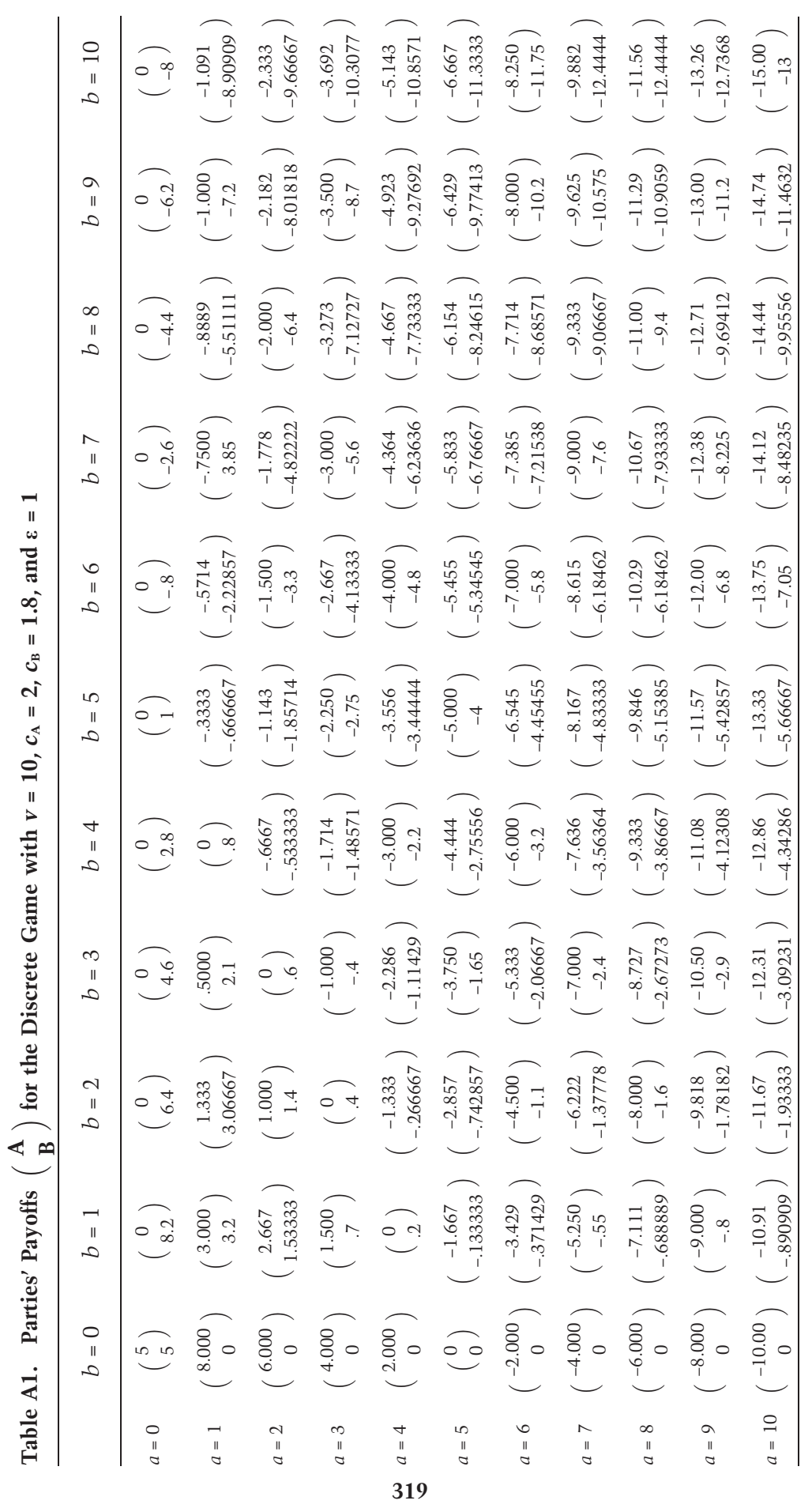




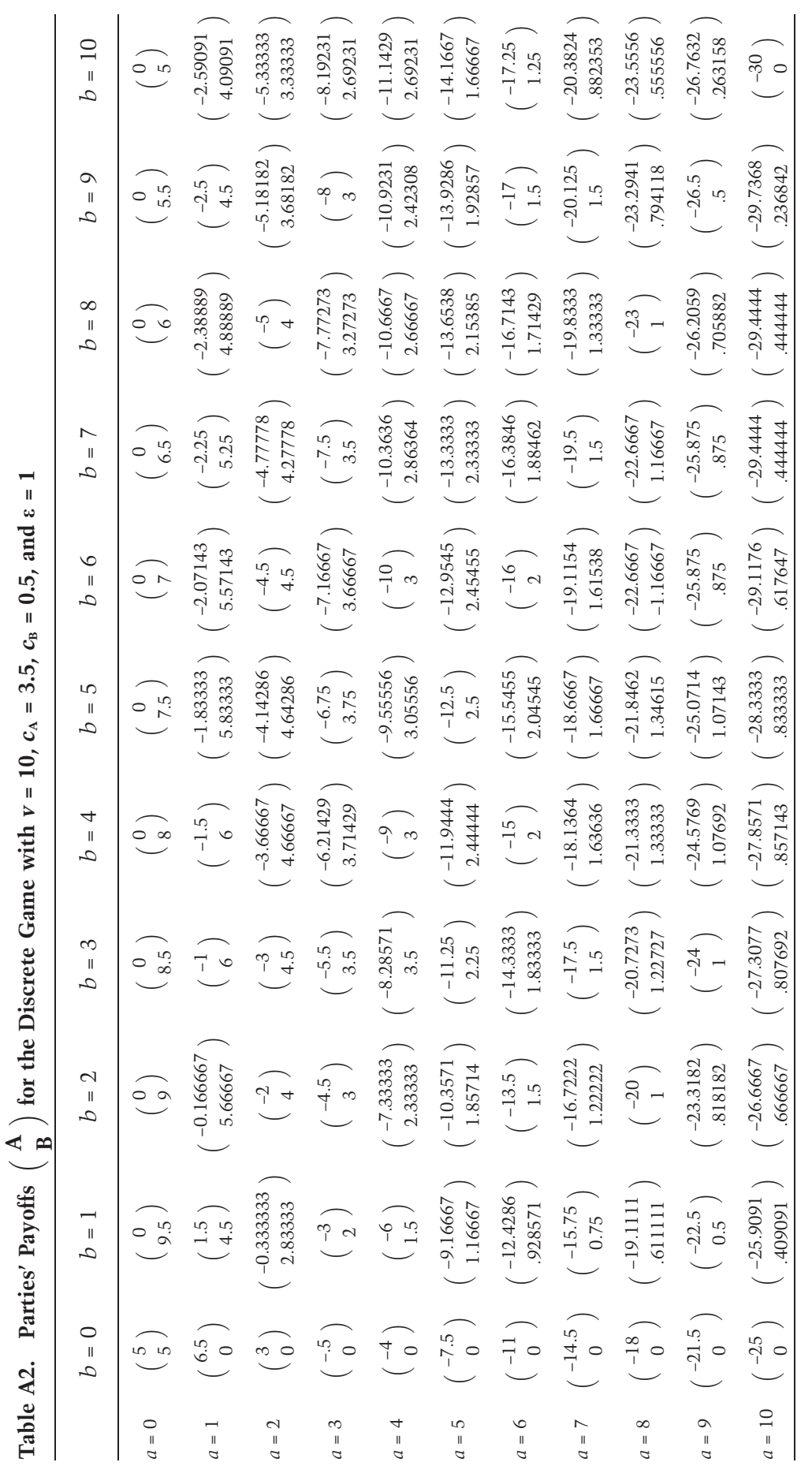




\section{R E F E R E N C E S}

Baye, Michael R., Dan Kovenock, and Casper G. Vries. 1994. "The Solution to the Tullock Rent-Seeking Game When R >2: MixedStrategy Equilibria and Mean Dissipation Rates." Public Choice 81 (3-4): 363-80.

Congleton, Roger D., and Arye L. Hillman, eds. 2015. Companion to the Political Economy of Rent Seeking. Cheltenham: Edward Elgar.

Congleton, Roger D., Arye L. Hillman, and Kai A. Konrad, eds. 2008a. 40 Years of Research on Rent Seeking 1. Theory of Rent Seeking. Berlin and Heidelberg: Springer.

Congleton, Roger D., Arye L. Hillman, and Kai A. Konrad, eds. 2008b. 40 Years of Research on Rent Seeking 2. Applications: Rent Seeking in Practice. Berlin and Heidelberg: Springer.

Dari-Mattiacci, Giuseppe, and Francesco Parisi. 2015. "Asymmetries in Rent-Seeking." In Companion to Political Economy of Rent Seeking, edited by Roger D. Congleton and Arye L. Hillman, 73-91. Cheltenham: Edward Elgar.

Krueger, Anne O. 1974. "The Political Economy of the Rent-Seeking Society." Journal of Political Economy 64 (3): 291-303.

Nash, John. 1951. "Non-Cooperative Games." Annals of Mathematics 54 (2): 286-95.

Tullock, Gordon. 1967. "The Welfare Costs of Tariffs, Monopolies, and Theft." Western Economic Journal 5 (3): 224-32.

Tullock, Gordon. 1980. "Efficient Rent Seeking." In Toward a Theory of the Rent-Seeking Society, edited by James M. Buchanan and Gordon Tullock, 97-112. College Station: Texas A\&M University Press. 